\title{
Aggrecan Degradation in Human Cartilage \\ Evidence for both Matrix Metalloproteinase and Aggrecanase Activity in Normal, Osteoarthritic, and Rheumatoid Joints
}

Michael W. Lark, ${ }^{*}$ Ellen K. Bayne, ${ }^{\star}$ Jamie Flanagan, ${ }^{\star}$ Coral F. Harper, ${ }^{*}$ Lori A. Hoerrner, ${ }^{*}$ Nancy I. Hutchinson, ${ }^{*}$ Irwin I. Singer,
Susan A. Donatelli, ${ }^{\star}$ Jeffrey R. Weidner, ${ }^{\star}$ Hollis R. Williams, ${ }^{*}$ Richard A. Mumford, ${ }^{\star}$ and L. Stefan Lohmander ${ }^{\ddagger}$
*Department of Inflammation Research, Merck Research Laboratories, Rahway, New Jersey 07065; and ${ }^{\ddagger}$ Department of Orthopedics,
University Hospital, 22185 Lund, Sweden

\section{Abstract}

To examine the activity of matrix metalloproteinases (MMPs) and aggrecanase in control and diseased human articular cartilage, metabolic fragments of aggrecan were detected with monospecific antipeptide antibodies. The distribution and quantity of MMP-generated aggrecan G1 fragments terminating in VDIPEN ${ }^{341}$ were compared with the distribution of aggrecanase-generated G1 fragments terminating in NITEGE ${ }^{373}$. Both types of G1 fragments were isolated from osteoarthritic cartilage. The sizes were consistent with a single enzymatic cleavage in the interglobular domain region, with no further proteolytic processing of these fragments. Both neoepitopes were also detected by immunohistochemistry in articular cartilage from patients undergoing joint replacement for osteoarthritis (OA), rheumatoid arthritis (RA), and in cartilage from adults with no known joint disease.

In control specimens, the staining intensity for both G1 fragments increased with age, with little staining in cartilage from 22-wk-old fetal samples. There was also an increase with age in the extracted amount of MMP-generated neoepitope in relation to both aggrecan and collagen content, confirming the immunohistochemical results. After the age of 20-30 yr this relationship remained at a steady state. The staining for the MMP-generated epitope was most marked in control cartilage exhibiting histological signs of damage, whereas intense staining for the aggrecanase-generated fragment was often noted in adult cartilage lacking overt histological damage. Intense staining for both neoepitopes appeared in the more severely fibrillated, superficial region of the tissue.

Intense immunostaining for both VDIPEN- and NITEGEneoepitopes was also detected in joint cartilage from patients with OA or RA. Cartilage in these specimens was significantly more degraded and high levels of staining for both epitopes was always seen in areas with extensive cartilage damage. The levels of extracted VDIPEN neoepitope relative to collagen or aggrecan in both $\mathrm{OA}$ and RA samples

Michael W. Lark and Ellen K. Bayne contributed equally to this work. Address correspondence to Michael W. Lark, SmithKline Beecham Pharmaceuticals, 709 Swedeland Road, uw2109, King of Prussia, PA 19406. Phone: 610-270-4914; FAX: 610-270-5598; E-mail: michael_ lark-1@sbphrd.com

Received for publication 2 December 1996 and accepted in revised form 8 April 1997.

J. Clin. Invest.

(C) The American Society for Clinical Investigation, Inc.

0021-9738/97/07/0093/14 \$2.00

Volume 100, Number 1, July 1997, 93-106 were similar to those seen in age-matched control specimens.

Immunostaining for both types of aggrecan fragments was seen surrounding the cells but also further removed in the interterritorial matrix. In some regions of the tissue, both neoepitopes were found while in others only one was detected. Thus, generation and/or turnover of these specific catabolic aggrecan fragments is not necessarily coordinated. Our results are consistent with the presence in both normal and arthritic joint cartilage of proteolytic activity against aggrecan based on both classical MMPs and "aggrecanase." (J. Clin. Invest. 1997. 100:93-106.) Key words: aggrecan • matrix metalloproteinases - aggrecanase - cartilage • osteoarthritis

\section{Introduction}

The destruction of joint cartilage is of central importance in human arthritic disease. Advances are now being made in our understanding of the events which initiate joint diseases, as well as of the processes involved in the degradation of articular cartilage in osteoarthritis $(\mathrm{OA})^{1}$ and RA. For example, in patients with RA and OA, there is clear evidence for increased expression and synthesis of enzymes, including matrix metalloproteinases (MMPs), in synovial tissue and cartilage, and of increased concentrations of the same proteases in joint fluids (1-9). These enzymes are synthesized as proenzymes and the majority of the enzyme that has been described in cartilage or joint fluid is inactive proenzyme $(1,5)$. This has left us with the question as to what role, if any, these enzymes play in cartilage matrix degradation in joint disease.

One of the initial approaches taken to address whether MMPs have the capacity to degrade matrix components in situ, was to identify and characterize specific enzyme-generated cleavage products in vitro (10-14). Cartilage extracts were prepared and the cleavage sites identified using $\mathrm{NH}_{2}$-terminal sequence analysis. Using this approach, evidence for MMP-generated degradation of link protein (10) and aggrecan (11) have been found. To further define the identity, distribution and quantity of such fragments, monospecific antibodies were produced that recognize enzyme-generated neoepitopes that are not present in the native, uncleaved molecules. The MMP-generated neoepitope in link protein was thus detected in young cartilage, and with age and joint disease there was a reduction in this epitope (15). These data suggested that MMP degradation of link protein may be involved in normal matrix turnover

1. Abbreviations used in this paper: G1 fragments, the amino-terminal, hyaluronan-binding globular domain of aggrecan; $\mathrm{GuHCl}$, guanidine hydrochloride; HA, hyaluronan; MMP, matrix metalloproteinase; OA, osteoarthritis. 
and that further catabolic processes may be involved in link protein degradation with age.

More recently a monoclonal antibody was generated against a neoepitope in type II collagen (16). This antibody does not detect native intact type II collagen but does detect cleaved collagen once the triple helix has unwound. With the antibody, it was shown that there is a regional elevation in this neoepitope that correlates with osteoarthritic disease severity as defined by histopathological score (16).

It has been reported that aggrecan G1 fragments increase in cartilage with age $(17,18)$. Stromelysin-1 (MMP-3), as well as other MMPs, cleave aggrecan in the interglobular domain between $\mathrm{Asn}^{341}$ and Phe ${ }^{342}$ to generate a G1 fragment with the COOH terminus VDIPEN ${ }^{341}$ (11-13). This fragment has been isolated and identified by $\mathrm{NH}_{2}$-terminal sequence analysis from human OA cartilage (11). A second proteolytic activity identified as "aggrecanase" also cleaves aggrecan in the interglobular domain, but between $\mathrm{Glu}^{373}$ and $\mathrm{Ala}^{374}$ (19-24), generating a $\mathrm{G} 1$ fragment with a $\mathrm{COOH}$ terminus of NITEGE ${ }^{373}$ (Please refer to Fig. 9 for a schematic representation of the $\mathrm{COOH}$ and $\mathrm{NH}_{2}$ termini thus generated in the aggrecan interglobular domain).

Large aggrecan fragments which have an $\mathrm{NH}_{2}$ terminus of ${ }^{374} \mathrm{ARGSV}$, consistent with aggrecanase cleavage, have been detected in human joint fluids by $\mathrm{NH}_{2}$-terminal sequence analysis $(25,26)$. However, no $\mathrm{G} 1$ fragments terminating in NITEGE ${ }^{373}$ have been identified in human cartilage to date. In contrast, it has been shown that the NITEGE-positive G1 fragment does accumulate in the culture medium of both retinoic acid and IL-1-stimulated rat chondrosarcoma cells without any accumulation of the VDIPEN-positive fragment (23). This indicates that aggrecanase-mediated aggrecan cleavage can take place independent of MMP-mediated degradation. The only isolated enzyme which has been shown to cleave between $\mathrm{Glu}^{373}$ and $\mathrm{Ala}^{374}$ is neutrophil collagenase (MMP-8) (27). However, this enzyme preferentially cleaves at the MMP site, between $\mathrm{Asn}^{341}$ and $\mathrm{Phe}^{342}$.

To define the distribution and quantity of these aggrecan G1 fragments in cartilage, we have developed antisera which detect either the MMP-generated G1 fragment (antiVDIPEN) (28) or the aggrecanase-generated G1 fragment (anti-NITEGE) (23). Neither antibody detects intact aggrecan $(23,28)$. In this study, we have used both the anti-VDIPEN and anti-NITEGE antisera to evaluate the distribution and quantities of these aggrecan-related epitopes in human articular cartilage. Specimens from patients undergoing joint replacement for OA or RA as well as specimens from individuals with no known joint disease were evaluated for the distribution of both neoepitopes. ${ }^{2}$

\section{Methods}

Materials. Materials were purchased from the following sources: benzamidine hydrochloride, 6 -amino- $n$-hexanoic acid, $N$-ethylmaleimide (NEM), PMSF, and papain from Sigma Chemical Co., St. Louis, MO; Guanidine hydrochloride $(\mathrm{GuHCl})$ from Pierce Chemical Co., Rockford, IL; EDTA and Alcian blue 8GX from Aldrich Chemical Co., Milwaukee, WI; protease-free chondroitinase ABC and keratanase II

2. Residue numbers throughout the manuscript are based on human sequence analysis (29) assuming that $\mathrm{Val}^{20}$ of that sequence is the $\mathrm{NH}_{2}$ terminus of the mature protein and is therefore numbered as residue $1\left[\mathrm{Val}^{1}\right]$. from Seikagaku Corp., Tokyo, Japan; L-Hydroxyproline from Eastman Kodak Co., Rochester, NY; dextran T-70 from Pharmacia, Uppsala, Sweden; charcoal from Mallinckrodt, Paris, KY; PBS from Gibco BRL, Grand Island, NY; SDS-polyacrylamide Tris-glycine gels from Integrated Separation Systems (ISS), Natick, MA; nitrocellulose membranes from Schleicher \& Schuell, Keene, NH; biotinylated goat anti-rabbit IgG $(\mathrm{H}+\mathrm{L})$, alkaline phosphatase streptavidin, and 5-bromo-4-chloro-3-indolyl-phosphate/nitroblue tetrazolium (BCIP/ NBT) substrate systems are from Kirkegaard \& Perry Laboratories (KPL), Gaithersburg, $\mathrm{MD} ; \mathrm{Na}^{125} \mathrm{I}$ from Amersham Corp., Arlington Heights, IL; avidin/biotin blocking kit and Vectastain Elite ABC kit, from Vector Laboratories, Inc., Burlingame, CA. Recombinant human stromelysin-1 was obtained from an expressing mammalian cell line generated by Celltech Ltd., Slough, United Kingdom.

Human cartilage samples. Samples were obtained from patients undergoing joint replacement surgery for OA or RA. Surgical samples were kept on ice and cartilage dissected from the tibial condyles within $1 \mathrm{~h}$ of surgery. For immunohistochemistry, full thickness joint cartilage samples were immersed in OCT embedding medium and snap frozen on liquid nitrogen. Where anatomy allowed, continuous cartilage samples were dissected from the periphery of the joint through the loaded area towards the tibial spines. Parallel samples for extraction were dissected and put into plastic vials and snap frozen in liquid nitrogen. All samples were stored at $-70^{\circ} \mathrm{C}$. For details on patient history, diagnosis, age and current medication, see Table I. Control cartilage was obtained at the time of lower extremity tumor resection from patients with no known joint disease, and processed as above. In no case were these patients treated with chemotherapy before surgery. Finally, cartilage was also obtained within $24 \mathrm{~h}$ of death from individuals with no known history of joint disease. Here, the tibial plateaus were collected by the International Institute for the Advancement of Medicine (71 West Uwchlan Avenue, Suite 120, Exton, PA 19341) and processed for either immunohistochemistry or radioimmunoassay. In some cases only one specimen per patient was prepared; however, in the majority of the cases, multiple specimens were taken from the cartilage of single individuals. This allowed for an analysis of the presence of epitope regionally within the joint as well as the ability to directly compare immunochemical quantitation with immunohistochemical analysis of parallel samples.

Extraction of human cartilage samples. Cartilage samples, $\sim 1 \mathrm{~mm}$ in cube, were extracted with a 20 -fold volume of $4.0 \mathrm{M}$ guanidine hydrochloride in a proteinase inhibitor cocktail consisting of $100 \mathrm{mM}$ 6-amino- $n$-hexanoic acid, $10 \mathrm{mM}$ ethylenediamine tetraacetic acid, 10 $\mathrm{mM}$ benzamidine hydrochloride, $100 \mathrm{mM} n$-ethylmaleimide, and 10 $\mathrm{mM}$ phenylmethyl sulfonyl fluoride at $4^{\circ} \mathrm{C}$ for $48 \mathrm{~h}$ (30). After extraction, the samples were centrifuged at $3,000 \mathrm{~g}$ for $10 \mathrm{~min}$ to separate the extracted collagen-rich cartilage pellet from the supernatant containing the proteoglycans and proteoglycan fragments. The collagen content was determined after acid hydrolysis of the pellet (31). The supernatant was removed and stored at $-70^{\circ} \mathrm{C}$ and used for quantitative assessment of VDIPEN by radioimmunoassay.

Isolation of aggrecan fragments for Western blotting, using cesium chloride density gradient centrifugation. The supernatant containing aggrecan and aggrecan fragments was brought to $50 \mu \mathrm{g} / \mathrm{ml}$ with human umbilical cord hyaluronan and dialyzed in a 3,000-kD cut-off membrane for $24 \mathrm{~h}$ at $4^{\circ} \mathrm{C}$ against $0.1 \mathrm{M}$ sodium acetate, $\mathrm{pH}$ 6.0, containing the proteinase inhibitor cocktail. After dialysis, the sample was clarified by centrifugation and the aggrecan-hyaluronan complex in the supernatant fractionated in an associative cesium chloride density gradient (starting density $1.5 \mathrm{grams} / \mathrm{ml}$ ). The bottom one-fourth of the gradient (A1) was harvested and brought to $4 \mathrm{M}$ with solid $\mathrm{GuHCl}$ and fractionated on a dissociative cesium chloride density gradient (starting density $1.5 \mathrm{grams} / \mathrm{ml}$ ). The top one fourth of this gradient (A1D4) was harvested, dialyzed against water, lyophilized, and evaluated by Western blotting using the anti-VDIPEN and antiNITEGE antisera $(23,28)$.

RIA to quantify VDIPEN neoepitope. The MMP-generated neoepitope, VDIPEN, was quantitated in the $4 \mathrm{M} \mathrm{GuHCl}$ extracts using 


\begin{tabular}{|c|c|c|c|c|c|c|}
\hline Patient & $\begin{array}{l}\text { Age at } \\
\text { surgery }\end{array}$ & History & Gender & Diagnosis & Radiologic findings & Medication at surgery \\
\hline & & $y r$ & & & & \\
\hline 1 & 56 & 10 & $\mathrm{~F}$ & RA & Almost complete loss of joint space & \\
\hline 2 & 72 & 24 & $\mathrm{~F}$ & RA & $\begin{array}{l}\text { Almost complete loss of joint space } \\
\text { medial + lateral compartment }\end{array}$ & $\begin{array}{l}\text { Indomethacin (previously chloroquine, } \\
\text { myocrisin, methotrexate) }\end{array}$ \\
\hline 3 & 67 & 7 & $\mathrm{~F}$ & Initially OA, subsequently RA & $\begin{array}{c}\text { Advanced with loss of joint space } \\
\text { medial + lateral compartment }\end{array}$ & Sulfasalazine, indomethacin \\
\hline 4 & 66 & 3 & $\mathrm{~F}$ & $\mathrm{OA}$ & Loss of $>50 \%$ of medial joint space & Diclofenac \\
\hline 5 & 78 & 10 & $\mathrm{~F}$ & $\mathrm{OA}$ & $\begin{array}{l}\text { Complete loss of medial joint space, } \\
\text { partial loss of lateral }\end{array}$ & Analgesics \\
\hline 6 & 78 & & M & RA & Almost complete loss of joint space & \\
\hline 7 & 65 & 8 & $\mathrm{~F}$ & $\begin{array}{l}\text { Secondary OA (post tibial } \\
\text { condyle fracture) }\end{array}$ & Complete loss of lateral joint space & Analgesics \\
\hline 8 & 84 & 5 & $\mathrm{~F}$ & $\mathrm{OA}$ & $\begin{array}{l}\text { Complete loss of medial joint space, } \\
\text { partial loss of lateral }\end{array}$ & \\
\hline 9 & 65 & 6 & M & $\mathrm{OA}$ & $\begin{array}{l}\text { Partial loss }(<50 \%) \text { of } \\
\text { medial joint space }\end{array}$ & \\
\hline 10 & 65 & 3 & $\mathrm{~F}$ & $\mathrm{OA}$ & Loss of $>50 \%$ of medial joint space & Naproxen \\
\hline 11 & 76 & 5 & M & $\mathrm{OA}$ & Loss of medial joint space & \\
\hline 12 & 64 & 11 & $\mathrm{~F}$ & $\mathrm{OA}$ & Loss of $>50 \%$ of lateral joint space & \\
\hline 13 & 59 & 11 & M & OA & $\begin{array}{l}\text { Almost complete loss of medial } \\
\text { joint space }\end{array}$ & \\
\hline 14 & 79 & 15 & M & RA & Complete loss of lateral joint space & Naproxen \\
\hline 15 & 84 & 8 & $\mathrm{~F}$ & $\mathrm{OA}$ & $\begin{array}{l}\text { Almost complete loss of medial } \\
\text { joint space }\end{array}$ & Analgesics \\
\hline 16 & 69 & 7 & $\mathrm{~F}$ & OA & Loss off $>50 \%$ of medial joint space & Analgesics \\
\hline 17 & 56 & 9 & $\mathrm{~F}$ & RA & Advanced joint destruction & $\begin{array}{l}\text { Corticosteroids + NSAIDs (previously } \\
\text { chloroquine, penicillamine, } \\
\text { myocrisin, sulfasalazine, } \\
\text { methotrexate, imurel) }\end{array}$ \\
\hline 18 & 82 & 37 & M & $\mathrm{OA}$ & Loss of medial joint space & \\
\hline 19 & 83 & & M & OA, prior tibial osteotomy & Loss of medial joint space & \\
\hline
\end{tabular}

the radioimmunoassay previously described (28). Briefly, cartilage extracts were initially diluted $1: 100$ into a total volume of $100 \mu \mathrm{l}$ of RIA buffer (PBS, $0.1 \%$ gelatin, $0.01 \%$ thimerasol, and $1 \mathrm{mM}$ ethylenediamine tetraacetic acid). At this concentration of $0.4 \mathrm{M} \mathrm{GuHCl}$ and proteinase inhibitors, there is no interference with neoepitope detection by the assay. The samples were incubated with $100 \mu \mathrm{l}$ of a 1:4,000 dilution of the rabbit anti-VDIPEN antiserum for $24 \mathrm{~h}$ at $4^{\circ} \mathrm{C}$. Samples were then incubated with $100 \mu \mathrm{l}$ of the ${ }^{125}$ I-YTGEDFVDIPEN peptide probe at $4^{\circ} \mathrm{C}$ for $24 \mathrm{~h}$. Unbound radiolabeled probe was removed from the solution by adding $1 \mathrm{ml}$ of dextran-coated charcoal for $10 \mathrm{~min}$ on ice followed by centrifugation at $900 \mathrm{~g}$ for 10 min. The supernatant was decanted and the amount of radioactivity determined in a gamma counter.

Stromelysin digestion of the cartilage extracts. Aliquots of the sample extracts were dialyzed against water, lyophilized, and reconstituted in a buffer containing $25 \mathrm{mM}$ Tris, $10 \mathrm{mM} \mathrm{CaCl}_{2}, 0.02 \%$ sodium azide, and $0.15 \mathrm{M} \mathrm{NaCl}$. A volume of $100 \mu \mathrm{l}$ of sample was incubated with $13 \mu \mathrm{g}$ of trypsin-activated recombinant human stromelysin- 1 for $24 \mathrm{~h}$ at $37^{\circ} \mathrm{C}$. The digestion was terminated with the addition of $10 \mathrm{mM}$ EDTA. The VDIPEN content was quantified in each of the samples using the RIA.

Immunohistochemical detection of neoepitopes. $6-\mu \mathrm{m}$ thick cryosections were cut on a model OTF Bright Cryotome (Hacker Instruments, Inc., Fairfield, NJ) equipped with a cryostat frozen sectioning aid (Instrumedics, Inc., Teaneck, NJ), mounted on glass slides and permeabilized by digestion with protease-free chondroitinase $\mathrm{ABC}$
(0.02 U/50 ml 0.1 M Tris- $\mathrm{HCl} \mathrm{pH} \mathrm{8.0)} \mathrm{for} 20 \mathrm{~min}$ at $37^{\circ} \mathrm{C}$. After washing in PBS, the sections were fixed with periodate-lysine-paraformaldehyde fixative (32) and treated with $3 \% \mathrm{H}_{2} \mathrm{O}_{2}$ in methanol to inactivate endogenous peroxidases, followed by treatment with $0.1 \%$ Triton-X 100 in PBS $\left(4^{\circ} \mathrm{C}\right.$ for $\left.5 \mathrm{~min}\right)$. Biotin and avidin binding sites within the sections were blocked with biotin and avidin solutions as specified in the vector blocking kit. Sections were then treated with preimmune, anti-VDIPEN or anti-NITEGE IgG and bound antibodies detected via immunoperoxidase microscopy using the avidin-biotin complex method. Peroxidase reaction product was developed with a glucose oxidase/diaminobenzidine/nickel method (33). A 1\% solution of eosin was used as a counterstain. For specificity controls the primary antibody was preincubated for $3 \mathrm{~h}$ at room temperature with 1 to $100-\mu \mathrm{mol}$ concentrations of synthetic peptide terminating with the neoepitope sequence or spanning the cleavage site (YTGEDFVDIPEN, YTGEDFVDIPENFFGV, YPLPRNITEGE, or YPLPRNITEGEAR) and clarified before use.

Parallel sections were stained with and without previous chondroitinase digestion and similar patterns of staining were observed. Chondroitinase digestion did, however, intensify the signal, and was therefore routinely used. Additional parallel sections of control and OA cartilages were stained with toluidine blue or hematoxylin and eosin and scored using the Mankin scoring system (34).

Stromelysin digestion of tissue sections. In some cases, as a positive control, unfixed cryosections were digested with trypsin-activated recombinant human stromelysin-1 $(100 \mu \mathrm{g} / \mathrm{ml}$ in $25 \mathrm{mM}$ Tris- 
$\mathrm{HCl}, 150 \mathrm{mM} \mathrm{NaCl}, 10 \mathrm{mM} \mathrm{CaCl}_{2}, 0.02 \% \mathrm{NaN}_{3}$ ) for $30 \mathrm{~min}$ at $37^{\circ} \mathrm{C}$. The tissue sections were then stained for the neoepitopes described above. Trypsin was removed from all activated stromelysin samples using soybean trypsin inhibitor-agarose (35). The only proteolytic activity remaining in the sample was due to stromelysin. This was confirmed by using transferrin as substrate, and showing that all proteolytic activity was inhibited by tissue inhibitor of metalloproteinases (TIMP) and 1,10-phenanthroline and not inhibited using PMSF. Further, second-order rate constants using a peptide substrate were shown to be similar to those published for stromelysin (36).

\section{Results}

Detection of aggrecan G1 fragments terminating in VDIPEN $N^{341}$ or NITEGE $E^{373}$ in extracts of human cartilage. To determine if aggrecan G1 fragments terminating with VDIPEN $^{341}$ or NITEGE ${ }^{373}$ were present within human cartilage, proteoglycans and proteoglycan fragments were extracted from cartilage from patients undergoing joint replacement surgery for OA. The extracts were fractionated to isolate hyaluronan-binding aggrecan fragments and evaluated on Western blots using the anti-VDIPEN and anti-NITEGE antisera (Fig. 1). In eight out of eight samples evaluated (Fig. 1 shows data for three of the samples), there were clearly separable $\sim 55 \mathrm{kD}$ VDIPEN-positive and $\sim 60 \mathrm{kD}$ NITEGE-positive G1 fragments that had maintained their ability to bind to hyaluronan, as indicated by their migration to the bottom of an associative cesium chloride density gradient. The VDIPEN-positive G1 fragment was similar in size to that generated by digestion of aggrecan with stromelysin in vitro (28), while the NITEGE-positive band migrated similarly to the G1 fragment generated by retinoic acid stimulated rat chondrosarcoma cells (23). The size of these fragments indicate that both full length MMP-generated $\left(\mathrm{Val}^{1}-\mathrm{Asn}^{341}\right)$ and aggrecanase-generated $\left(\mathrm{Val}^{1}-\mathrm{Glu}^{373}\right)$ aggrecan G1-fragments are present in human OA cartilage.

Immunohistochemical staining for the VDIPEN and NITEGE neoepitopes in control human cartilage. To evaluate the distribution of VDIPEN and NITEGE neoepitopes, im-
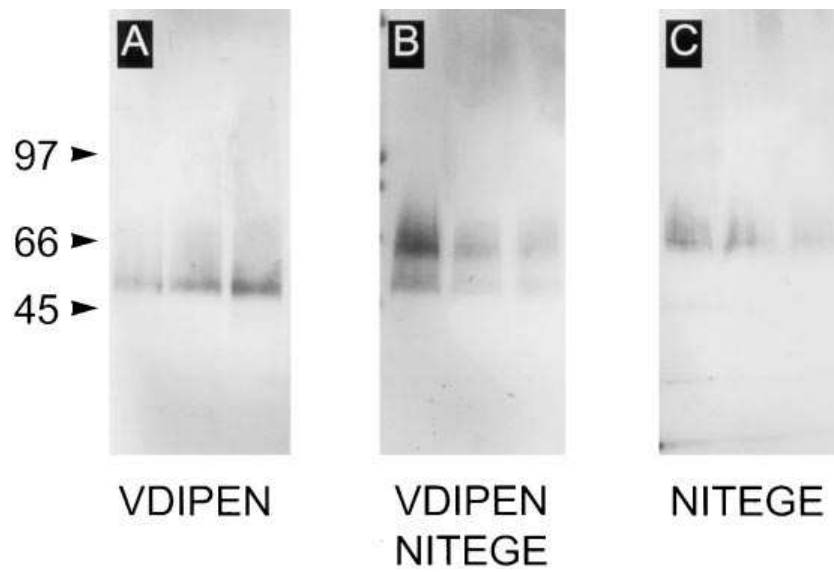

Figure 1. Western blot analysis of OA cartilage extracts. Extracts were prepared from cartilage of three patients (Tables II and III, samples 4 and 19; lane 3 is an additional OA sample not listed in the tables) undergoing joint replacement surgery for OA. Extracts were fractionated on associative and dissociative cesium chloride density gradients. The A1D4 fractions from the gradients were evaluated on Western blots probed with the $(A)$ anti-VDIPEN, $(B)$ anti-VDIPEN and anti-NITEGE, or $(C)$ anti-NITEGE antisera. munolocalization studies were carried out on cartilage specimens from subjects with no history of joint disease (ages $30 \mathrm{~d}$ to $72 \mathrm{yr}$ ). The pattern of immunoreactivity was compared to the degree of cartilage damage as assessed using the Mankin histopathological score (34). Cartilage from fetuses at 22 and $25 \mathrm{wk}$ of gestation was also examined. The neoepitope staining in these control cartilages was then compared with the staining patterns in cartilage specimens from individuals with OA or RA.

In fetal knee joint cartilage (Fig. $2 A$ ), and in cartilage from a 30-d-old subject (data not shown), staining for VDIPEN was limited to regions immediately adjacent to blood vessels. Little or no immunostaining was present elsewhere within the cartilage matrix. Very intense staining for the neoepitope could, however, be generated in the matrix by digestion of the frozen section with stromelysin-1 (data not shown), indicating that there is a significant amount of aggrecan within the matrix available for MMP cleavage. Unlike the fetal and newborn specimens, when cartilage from subjects $\geq 12 \mathrm{yr}$ (Fig. $2 C$ ) was examined, endogenous VDIPEN was readily detectable by immunostaining. In general, staining intensity appeared to increase with age as well as with the degree of histological damage. In undamaged cartilage from young adults (Fig. $2 E$ ), the neoepitope was usually most abundant towards the articular surface of the cartilage, where it was present throughout the matrix. In this region, as well as deeper within the cartilage, the most intense staining was localized in the pericellular matrix, with less intense staining in matrix further from the cells. Upon examination of cartilage specimens from progressively older subjects, as exemplified in a specimen from a 72-yr-old with no known joint disease (Fig. $2 G$ ), there was a notable increase both in the amount of immunostaining for VDIPEN and the amount of histological damage as assessed by histopathology scoring (Fig. 3) (34). The most intense staining for the neoepitope co-localized with the grossly damaged, fibrillated zone of the remaining articular cartilage. Staining was visible deeper in the cartilage where, however, it extended throughout the matrix but often appeared to be most concentrated pericellularly.

Immunostaining for the aggrecanase-generated neoepitope, NITEGE, was also detected in these specimens (Fig. 2, $B, D, F$, and $H)$. Although the distributions of the two neoepitopes overlapped in many regions, each of the neoepitopes extended into matrix in which the other was not detectable. In fetal knee joints, NITEGE, unlike VDIPEN, was detectable in superficial cartilage and meniscus (Fig. 2 B). Like VDIPEN, NITEGE was also concentrated around blood vessels. With age, NITEGE-staining at the articular surface became increasingly prominent. Young adult joint cartilage, though showing no overt histological damage, exhibited a wide band of very intense immunostaining for the NITEGE neoepitope in the superficial region of the cartilage (Fig. $2 F$ ). This band of immunostaining at the articular surface of the cartilage was much more prominent in NITEGE-stained sections than in adjacent VDIPEN-stained sections (Fig. 2, $F$ vs $E$ ). Beneath the region of intense NITEGE immunostaining, the neoepitope was much less abundant and detectable only in pericellular matrix. Staining for VDIPEN neoepitope was detectable throughout the matrix in deeper regions of the cartilage (Fig. 2 E). In cartilage specimens from older subjects with extensive damage as detected by histology, a similar pattern was observed for the two neoepitopes with a band of intense staining present at the damaged articular surface (Fig. 2, $G$ and $H$ ). 


\section{VDIPEN}
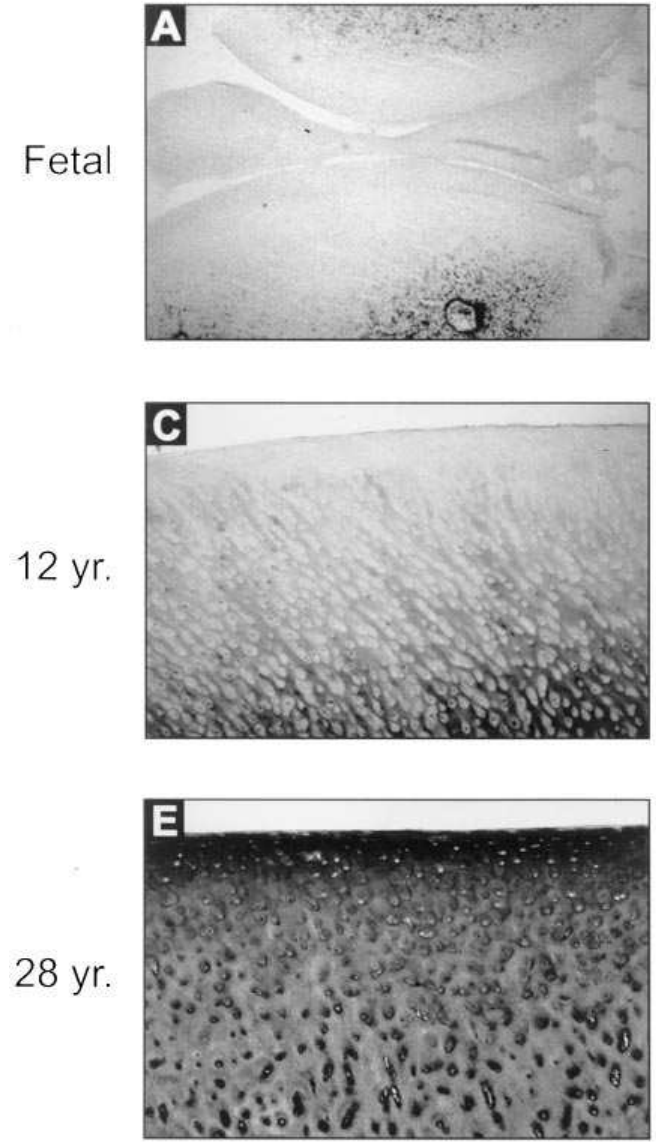

$72 \mathrm{yr}$.

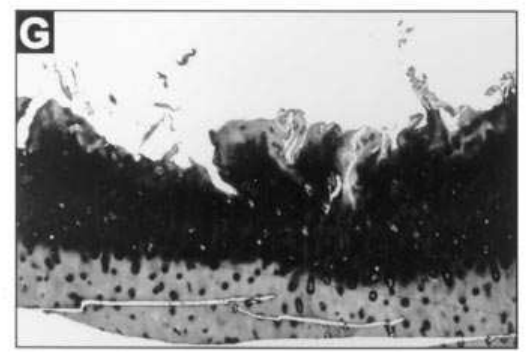

\section{NITEGE}
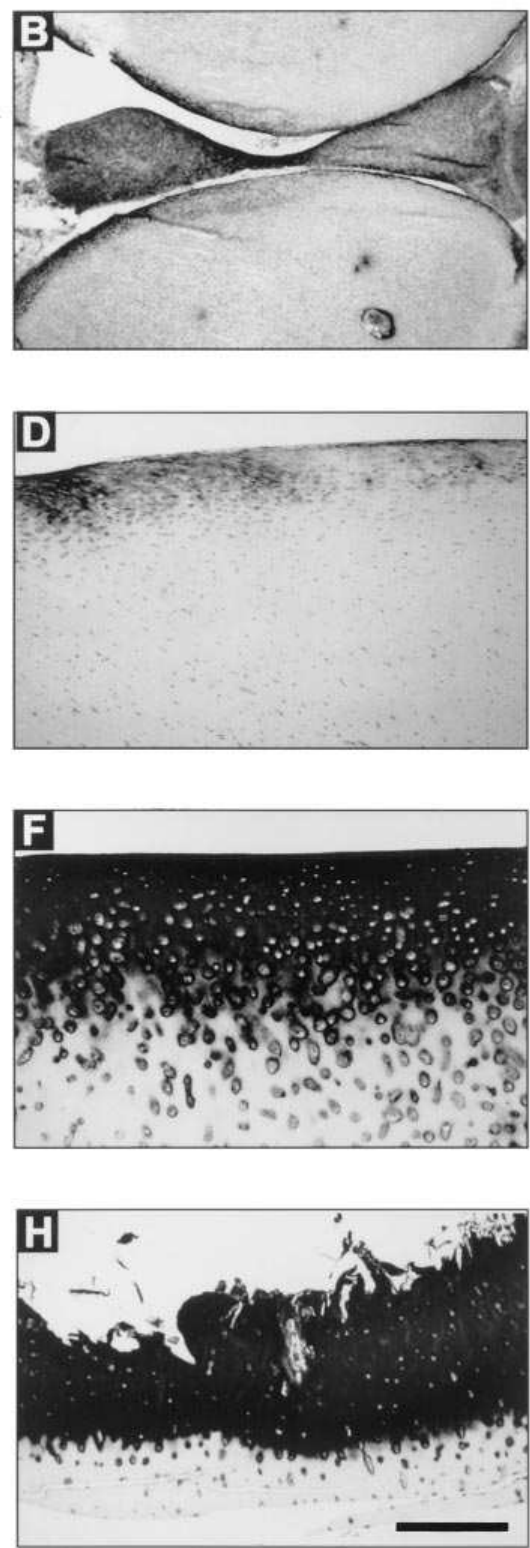

Figure 2. Immunoperoxidase labeling of VDIPEN $(A, C, E$, and $G)$ or NITEGE $(B, D, F, H)$ in cartilage from control subjects with no known history of joint disease. ( $A$ and $B$ ) Knee joint from a fetus at 22 wk gestation (Tables II and III, sample A) (femoral condyle top of micrograph, tibial condyle bottom). (C,D,E.F, $G$, and $H$ ) Cartilage from the tibial condyles of a 12-yr-old (Tables II and III, sample $G$ ), a 28-yr-old (Tables II and III, sample $H$ ), and a 72-year-old (Tables II and III, sample $Q)$, respectively. Bar $=600 \mu \mathrm{m}$.
Immunostaining for neoepitopes in $O A$ and $R A$ cartilage. Intense immunostaining for both VDIPEN- and NITEGEneoepitopes was detected in cartilage from patients with $\mathrm{OA}$ (Fig. 4, $A$ and $B$ ) and RA (Fig. 4, $C$ and $D$ ). Histological damage was much more extensive in these specimens and, as was the case in control samples, high levels of staining for both epitopes was always seen in areas with extensive cartilage damage. While the staining patterns observed for the two neoepitopes were often quite similar, it again was clear that the epitopes did not always codistribute. Both VDIPEN (Fig. 4, $A$ and $C$ ) and NITEGE (Fig. 4, $B$ and $D$ ) were most concentrated toward the articular surface of the cartilage where they were present in all matrix zones. Staining for VDIPEN, however, was frequently reduced or absent from the most distal tips of fibrillated strands of cartilage (Fig. 4, $A$ and $C$ ), while intense staining for NITEGE usually remained in these re- gions (Fig. 4, $B$ and $D$ ). In deeper cartilage, the intensity of immunostaining was reduced for both epitopes. VDIPEN staining, however, appeared to be more extensive in these regions than staining for NITEGE and often extended into areas with no detectable NITEGE.

Antibody specificity controls. The antibodies were preincubated with the antigen neoepitope peptides or with peptides spanning the cleavage sites (Fig. 5). In both cases, staining could be completely blocked by the appropriate peptide immunogen (Fig. 5, $C$ and $D$ as compared with $A$ and $B$, respectively), whereas the peptides spanning the cleavage sites had no effect on staining (Fig. 5, $E$ and $F$ as compared with $A$ and $B$, respectively). No immunostaining of any of the cartilage specimens was obtained when nonimmune rabbit IgG was substituted for anti-VDIPEN or anti-NITEGE IgG (data not shown). Together these data indicate that the anti-neoepitope 


\section{Relation between Histopathological Score and VDIPEN Staining Intensity in Human Knee Joint Cartilage}

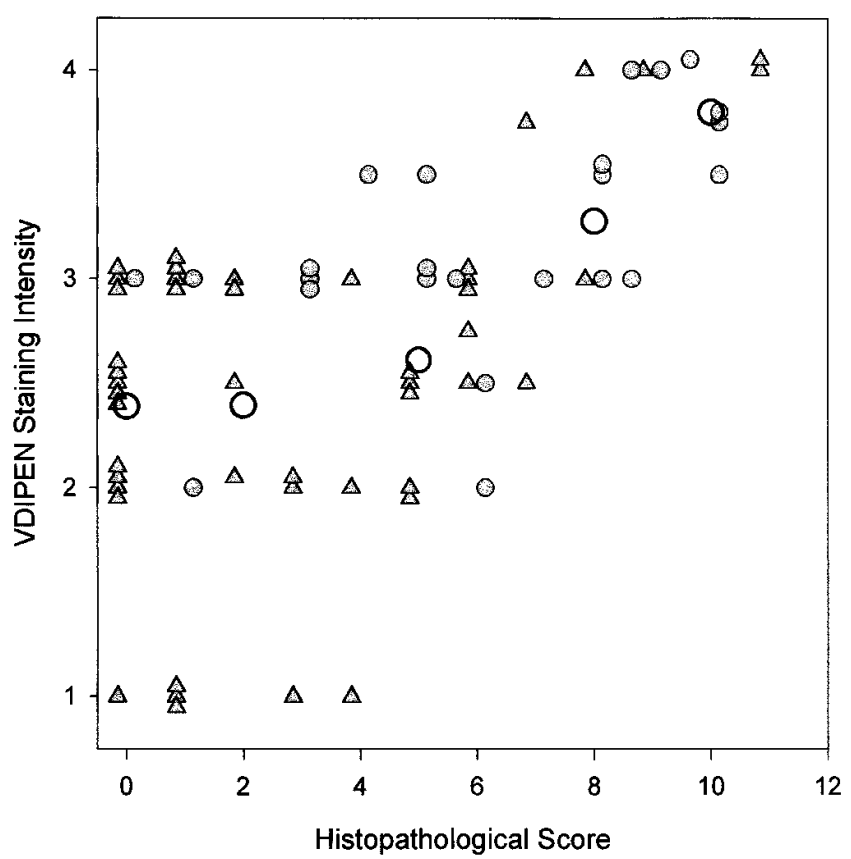

Figure 3. Relationship between intensity of immunostaining for VDIPEN neoepitope and degree of histopathology of joint cartilage from control and OA subjects. Intensity of immunostaining was scored visually on a scale of 1 to 4 with $1=$ very little staining, $2=$ low levels of staining, $3=$ moderate staining, and $4=$ high levels of staining. Histopathologic scoring of joint cartilage was done according to Mankin et al. (34). Filled circles represent samples from patients with diagnosed OA; filled triangles represent samples from subjects with no known joint disease; open circles represent median VDIPEN staining intensity for Mankin grades $0,1-3,4-6,7-8$ and $9-11$, respectively. A total of 71 sections were evaluated from 9 joints antibodies specifically detect the new $\mathrm{COOH}$ termini of the aggrecan G1 fragments localized within the cartilage.

Detection of neoepitopes in cartilage in the central weightbearing region in relation to the peripheral, nonweight-bearing region of cartilage. We evaluated the distribution of the neoepitopes in the cartilage normally covered by the meniscus in relation to the central weight-bearing region of the joint, using extended segments of cartilage obtained from joints of individuals with only minor joint damage. Because of the extensive damage and loss of articular cartilage in specimens from patients undergoing joint replacement for RA and OA, a similar analysis could not be completed on these samples. In the cartilage normally covered by meniscus, there was marked staining for both neoepitopes throughout the matrix in the most superficial region of the tissue (Fig. 6, $A$ and $B$ ). Deeper within the cartilage, there was significant interterritorial staining for both epitopes while the territorial and pericellular zones immediately surrounding the chondrocytes were devoid of staining. This is not due to these zones lacking aggrecan since treatment of the tissue sections with stromelysin-1 generates intense staining for the VDIPEN neoepitope in these areas (data not shown).

Staining for both neoepitopes was also present in the central weight-bearing region of the cartilage (Fig. 6, $C$ and $D$ ). VDIPEN neoepitope staining was most concentrated within the pericellular zone but extended throughout the territorial and interterritorial matrix. This staining pattern was seen throughout the full thickness of the cartilage. In contrast, immunostaining for NITEGE appeared as a prominent band at the articular surface of the cartilage, extending throughout all

of OA patients aged 59-84 yr, and 13 joints of control subjects aged 12-72 yr. For clarity, some data points have been slightly offset on the horizontal axis.
VDIPEN

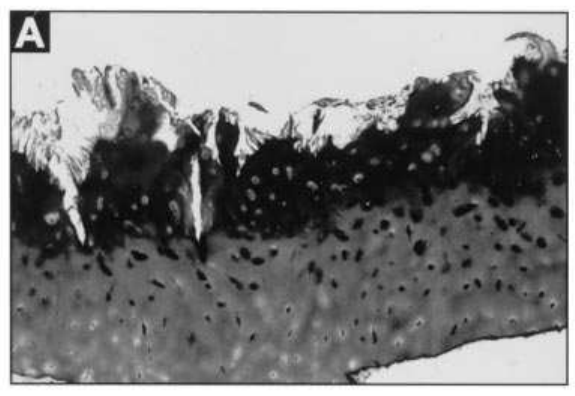

RA

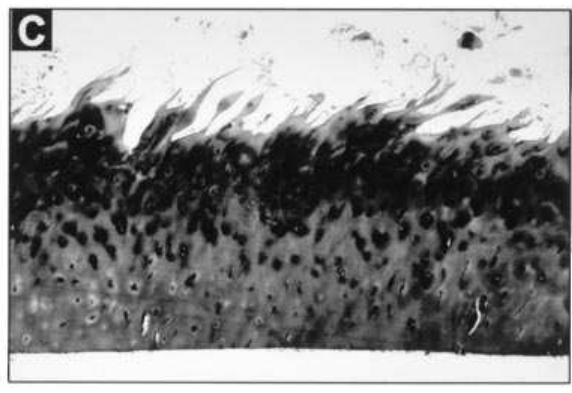

NITEGE
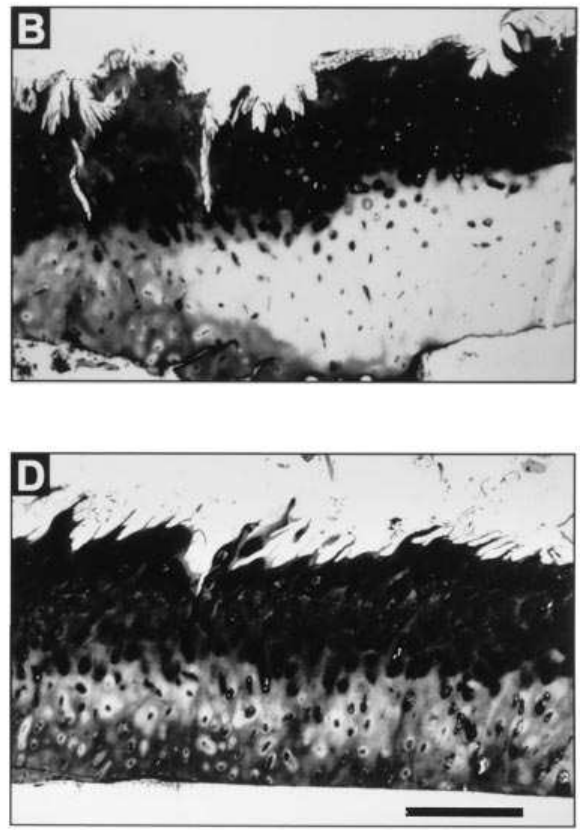

Figure 4. Immunoperoxidase labeling of VDIPEN $(A$ and $B)$ or NITEGE $(C$ and $D)$ in OA or RA cartilage. ( $A$ and $B$ ) Cartilage from the tibial condyle of a 69-yr-old patient with OA (Table I, sample 16). ( $C$ and $D$ ) Cartilage from the tibial condyle of a 79-yr-old patient with RA (Table I, sample 14). Bar $=600 \mu \mathrm{m}$. 


\section{VDIPEN}
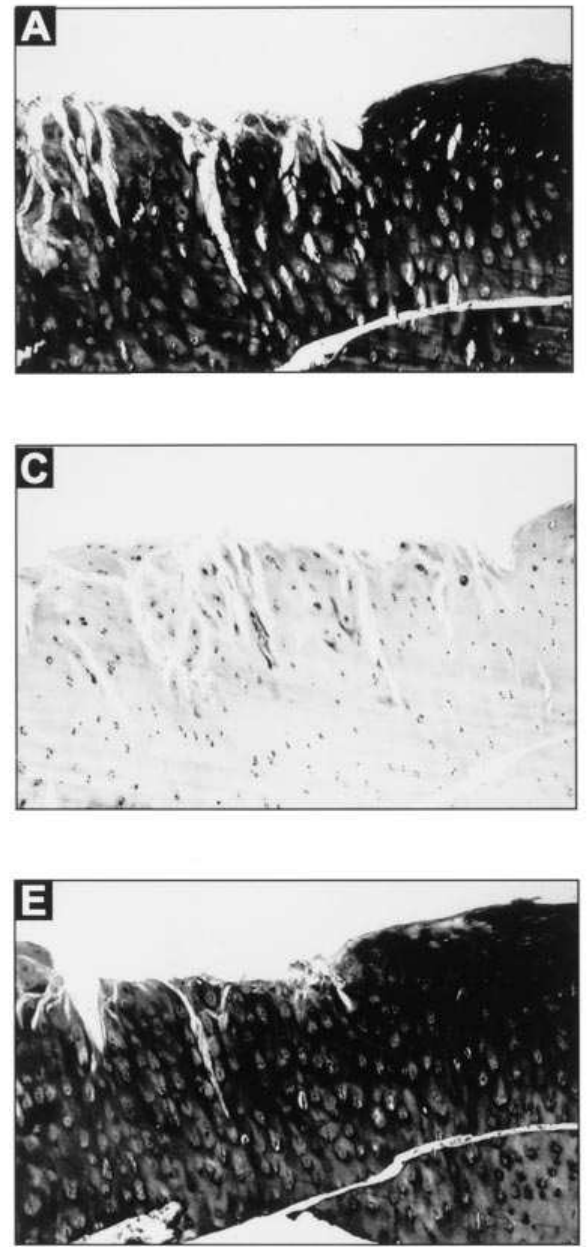

NITEGE
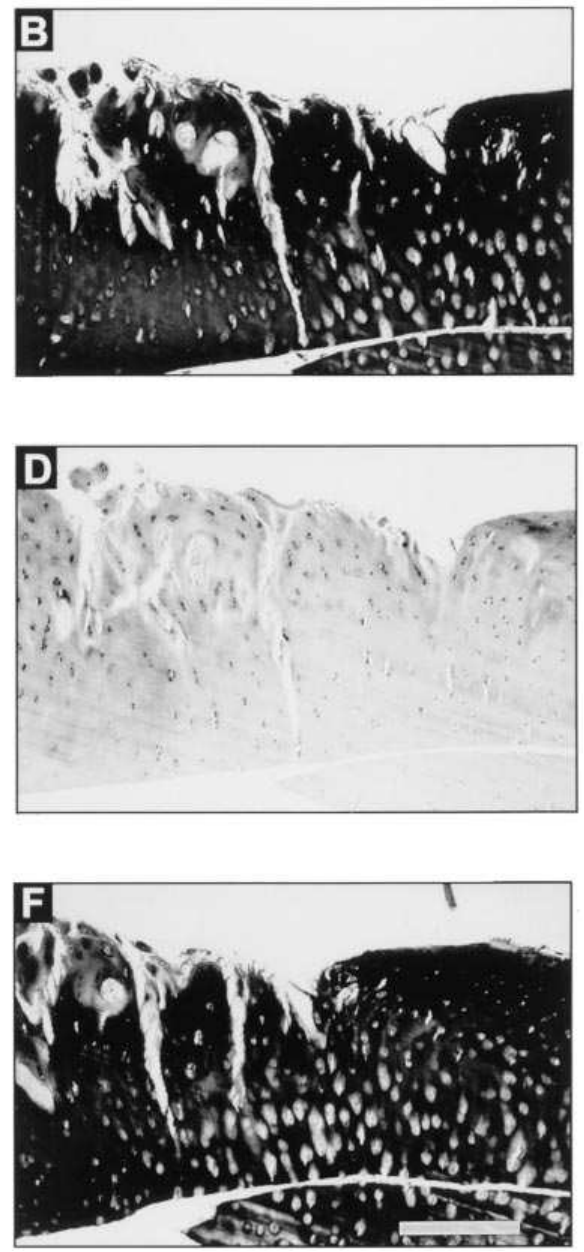

Figure 5. Immunoperoxidase labeling of VDIPEN or NITEGE in cryosections of cartilage from a 64-yr-old patient with OA (Table I, sample 12). Immunostaining for VDIPEN $(A)$ is completely abolished when antiVDIPEN IgG is preincubated with YTGEDFVDIPEN peptide $(C)$, but is unaffected by preincubation of the antibody with YTGEDFVDIPEN peptide $(E)$, a peptide spanning the metalloprotease cleavage site. Immunostaining for NITEGE $(B)$ is completely blocked by preincubation of anti-NITEGE IgG with YPLPRNITEGE peptide $(D)$, but is unaffected by preincubation with YPLPRNITEGEAR $(F)$, a peptide spanning the aggrecanase cleavage site. Bar $=600 \mu \mathrm{m}$ matrix zones. In deeper regions of the cartilage there was very little immunostaining for NITEGE and this was limited to the pericellular zone.

Specimens closer to the tibial spine showed a band of intense immunostaining for the NITEGE neoepitope extending in from the articular surface of the cartilage (Fig. $6 F$ ). Beneath this, immunostaining for NITEGE was concentrated in the pericellular matrix, with little or no staining in the territorial or interterritorial matrix. Staining for VDIPEN, on the other hand, did not appear to be preferentially concentrated toward the articular surface of the cartilage (Fig. $6 \mathrm{E}$ ). Again, the distribution of this neoepitope was similar throughout the thickness of the cartilage, with immunostaining most abundant in pericellular matrix. Together these data indicate that both neoepitopes are expressed throughout the articular cartilage, but that the patterns differ regionally.

The digestion of frozen tissue sections with stromelysin-1 increased the staining intensity for the VDIPEN neoepitope. Notably, however, the staining intensity for the NITEGE neoepitope was neither increased, nor abolished after digestion of frozen sections with the protease (data not shown). Loss of the NITEGE staining would be expected if this fragment could be further processed by MMPs such as stromelysin and the processed $\mathrm{COOH}$-terminal 32 amino acid fragment $\mathrm{Phe}^{342}$-Glu ${ }^{373}$ was released from the cartilage (see Figs. 9 and 10).

Quantitation of the VDIPEN neoepitope in control specimens of human cartilage. Initially, we characterized the quantity of the neoepitope in a number of control samples and then compared these to extracts of OA and RA cartilage. A confounding factor in our analysis is that we quantified neoepitope in extracts of full-thickness specimens, whereas the immunohistochemical evaluation allowed us to observe the differential distribution of the neoepitopes at the microscopic level throughout the thickness of the cartilage. We were able to quantify the VDIPEN neoepitope, since we have previously shown, using the RIA, that $1 \mathrm{~mol}$ of peptide equals $1 \mathrm{~mol}$ of VDIPEN-positive G1 fragment (28). Unfortunately, a similar quantitation of NITEGE-positive aggrecan fragments could not be completed because there is currently no reproducible way to generate an accurate G1-standard for this RIA (23).

18 cartilage specimens from thirteen individuals, ranging in age from $30 \mathrm{~d}$ to $88 \mathrm{yr}$, were obtained at the time of autopsy. Multiple samples were taken from these specimens and the VDIPEN neoepitope concentrations determined (Table II). The concentration of neoepitope in these samples ranged from 0.011 to 0.65 pmol VDIPEN per microgram hydroxyproline. 

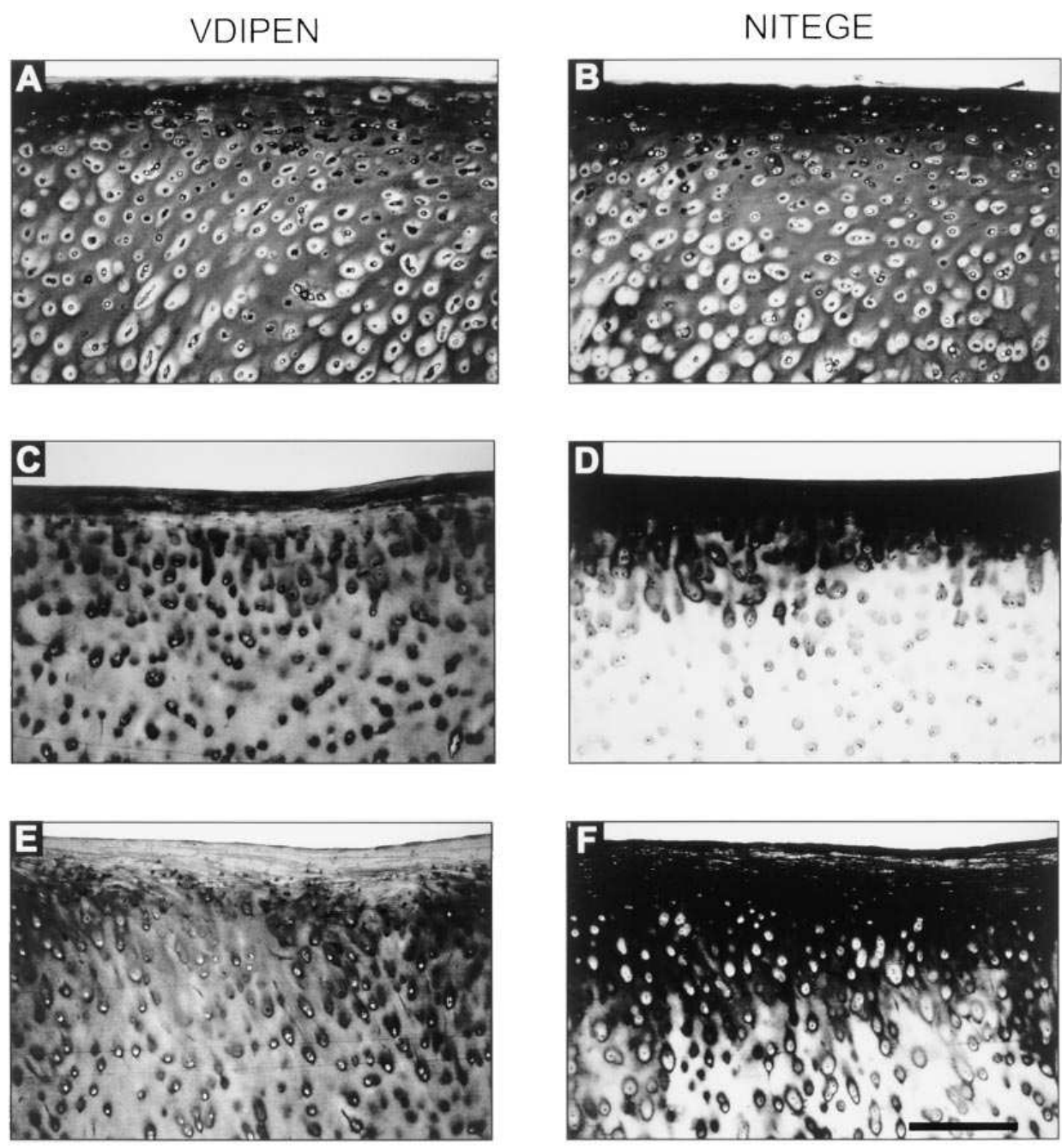

Figure 6. Cartilage from the tibial condyle of a 29-yr-old allograft donor (not in table) was cryosectioned and immunostained for VDIPEN $(A, C$, and $E)$ or NITEGE $(B, D$, and $F)$. ( $A$ and $B)$ Cartilage from an area that had been covered by meniscus. ( $C$ and $D$ ) Cartilage from a central, loaded, area not covered by the meniscus. ( $E$ and $F$ ) Cartilage from an area close to the tibial spines. Bar $=300 \mu \mathrm{m}$

pmol VDIPEN per $\mu \mathrm{g}$ OHpro in Human Control Cartilage

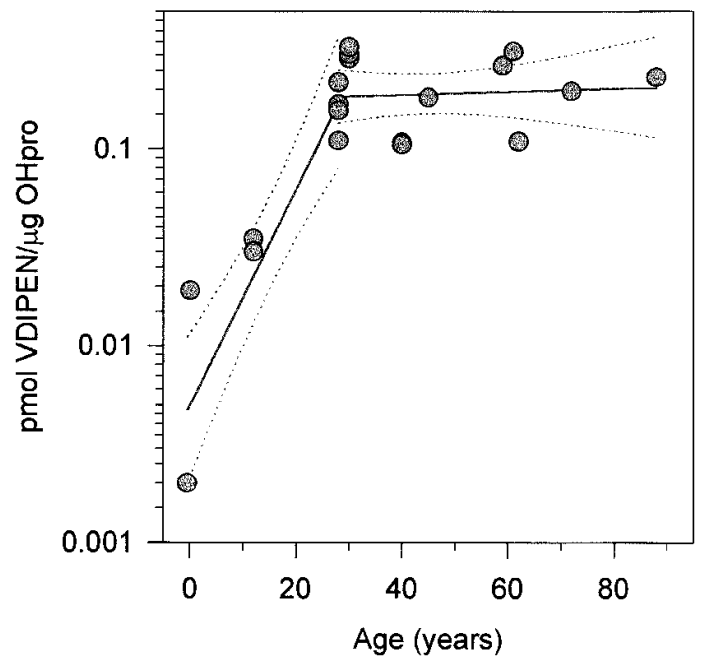

Figure 7. VDIPEN neoepitope ( $\mathrm{pmol}$ ) per microgram hydroxyproline in extracts of human joint cartilage from control patients with no history of joint disease. Results are plotted in relation to patient age. A first order regression line with $95 \%$ confidence interval (dotted
The concentration of VDIPEN neoepitope relative to collagen increased with age up to $\sim 25 \mathrm{yr}$, then reached an apparent steady state (Fig. 7). Extractability of aggrecan ranged from $75-90 \%$, with no relation to age. Therefore, the differences detected with age were not the result of altered extractability of the samples.

In specimens obtained from two fetuses with gestational ages of $22 \mathrm{wk}$, there was $<0.002$ pmol VDIPEN per microgram hydroxyproline. This was not the result of a lack of available aggrecan substrate, since VDIPEN-positive G1 fragments could be generated and detected by RIA and Western blot when the samples were incubated with stromelysin-1 (data not shown).

When the fetal knee cartilages from five specimens ranging in gestational age from 19-22 wk were reevaluated after stromelysin digestion, there were at least $900 \mathrm{~mol}$ of aggrecan that could be cleaved by stromelysin at the MMP site for every mole of resident VDIPEN-positive fragment. In fact, the relative increase in neoepitope ranged from 979- to over 13,172-fold with a median increase of 3,219-fold (Table III). Interestingly,

lines) is plotted for the ages 0 to $28 \mathrm{yr}$ and $>28 \mathrm{yr}$, respectively. Note logarithmic Y-axis. 
Table II. Quantitation of VDIPEN in Relation to Hydroxyproline Content in Human Knee Joint Cartilage Samples

\begin{tabular}{|c|c|c|c|c|c|}
\hline Sample patient & Age & Median & Range & $n$ & Diagnosis \\
\hline & $y r$ & 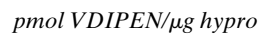 & & & \\
\hline A & 22-wk gestation & 0.002 & & 1 & $\mathrm{C}$ \\
\hline B & 22-wk gestation & 0.002 & & 1 & $\mathrm{C}$ \\
\hline $\mathrm{F}$ & $30 \mathrm{~d}$ & 0.019 & $0.011-0.038$ & 5 & $\mathrm{C}$ \\
\hline G1 & 12 & 0.035 & $0.029-0.056$ & 8 & $\mathrm{C}$ \\
\hline $\mathrm{G} 2$ & 12 & 0.030 & $0.025-0.056$ & 9 & $\mathrm{C}$ \\
\hline $\mathrm{H} 1$ & 28 & 0.110 & $0.070-0.276$ & 8 & $\mathrm{C}$ \\
\hline H1 (femur) & 28 & 0.168 & $0.030-0.257$ & 8 & $\mathrm{C}$ \\
\hline $\mathrm{H} 2$ & 28 & 0.156 & $0.088-0.282$ & 7 & $\mathrm{C}$ \\
\hline $\mathrm{H} 2$ (femur) & 28 & 0.217 & $0.038-0.302$ & 6 & $\mathrm{C}$ \\
\hline I & 29 & 0.288 & $0.237-0.315$ & 3 & $\mathrm{CT}$ \\
\hline $\mathrm{J}$ (right) & 30 & 0.303 & $0.081-0.435$ & 6 & $\mathrm{C}$ \\
\hline $\mathrm{J}(\mathrm{left})$ & 30 & 0.329 & $0.212-0.593$ & 7 & $\mathrm{C}$ \\
\hline $\mathrm{K}$ & 40 & 0.107 & $0.094-0.119$ & 2 & $\mathrm{CT}$ \\
\hline $\mathrm{L}$ & 40 & 0.105 & & 1 & $\mathrm{C}$ \\
\hline M & 45 & 0.182 & $0.108-0.650$ & 8 & $\mathrm{C}$ \\
\hline $\mathrm{N}$ & 59 & 0.264 & & 1 & $\mathrm{C}$ \\
\hline $\mathrm{O}$ & 61 & 0.312 & $0.200-0.423$ & 2 & $\mathrm{CT}$ \\
\hline $\mathrm{P}$ & 62 & 0.109 & $0.035-0.325$ & 11 & $\mathrm{C}$ \\
\hline Q & 72 & 0.196 & $0.071-0.242$ & 8 & $\mathrm{C}$ \\
\hline $\mathrm{R}$ & 88 & 0.231 & & 1 & $\mathrm{C}$ \\
\hline 17 & 56 & 0.112 & $0.091-0.133$ & 2 & RA \\
\hline 4 & 59 & 0.163 & & 1 & $\mathrm{OA}$ \\
\hline 13 & 60 & 0.331 & $0.105-0.556$ & 2 & $\mathrm{OA}$ \\
\hline 12 & 64 & 0.419 & $0.303-0.534$ & 2 & $\mathrm{OA}$ \\
\hline 7 & 65 & 0.109 & & 1 & $\mathrm{OA}$ \\
\hline 9 & 65 & 0.157 & $0.125-0.187$ & 2 & $\mathrm{OA}$ \\
\hline 10 & 65 & 0.342 & $0.322-0.361$ & 2 & $\mathrm{OA}$ \\
\hline 1 & 66 & 0.022 & & 1 & RA \\
\hline 3 & 67 & 0.310 & & 1 & RA \\
\hline 16 & 69 & 0.203 & $0.093-0.214$ & 3 & $\mathrm{OA}$ \\
\hline 2 & 71 & 0.146 & & 1 & RA \\
\hline 11 & 76 & 0.687 & $0.213-1.154$ & 3 & $\mathrm{OA}$ \\
\hline 5 & 78 & 0.140 & & 1 & $\mathrm{OA}$ \\
\hline 6 & 78 & 0.211 & $0.077-0.215$ & 3 & RA \\
\hline 14 & 79 & 0.310 & & 1 & RA \\
\hline 18 & 82 & 0.191 & & 1 & $\mathrm{OA}$ \\
\hline 19 & 83 & 0.129 & & 1 & $\mathrm{OA}$ \\
\hline 8 & 84 & 0.408 & $0.324-0.433$ & 3 & $\mathrm{OA}$ \\
\hline 15 & 84 & 0.087 & $0.078-0.095$ & 2 & $\mathrm{OA}$ \\
\hline
\end{tabular}

Extracts of human knee joint cartilage (Diagnosis: C, control; CT, Control from tumor case) were prepared and the VDIPEN neoepitope quantified by RIA. The collagen content in the collagen-rich pellet was then determined using a hydroxyproline assay and the data reported as picomoles VDIPEN/microgram hydroxyproline. $n$ denotes the number of samples examined from each joint. Unless noted, all samples were from tibial condyles. Control samples were numbered A-R, for numbering and further information on OA and RA patient samples, see Table I.

there were only $\sim 20 \mathrm{~mol}$ (range 16-37) of available substrate per mole of resident VDIPEN-positive G1 fragment in the samples from the 12-yr-old subject. When the remaining control samples were examined as a group (age range 28-88), there were from 3- to 25 -fold increases in signal after stromelysin digestion with a median increase of about eightfold (Table III). Again, it appeared that in the matrix the ratio of VDIPEN-positive G1 fragments to the available aggrecan substrate increased up to age 20-30, then remained at a steady state with increasing age (Fig. 8).
Together, these data suggest that the VDIPEN-positive G1 fragments are found at low levels in cartilage from very young individuals in relation to both collagen and available aggrecan substrate, but that this G1 fragment accumulates with age up to about $25 \mathrm{yr}$.

Quantitation of VDIPEN neoepitope in human $O A$ and $R A$ cartilage specimens. There was little difference in the average levels of FVDIPEN neoepitope relative to collagen in either the adult control samples $(0.205 \pm 0.08 \mathrm{pmol} / \mu \mathrm{g}$ hypro $[n=15$ median levels $])$, OA samples $(0.274 \pm 0.177 \mathrm{pmol} / \mu \mathrm{g}$ hypro $[n=$ 
Table III. VDIPEN Neoepitope in Human Knee Joint Cartilage Relative to Uncleaved Aggrecan Substrate (Expressed as Fold Increase after Stromelysin Digestion)

\begin{tabular}{|c|c|c|c|c|c|}
\hline Sample patient & Age & Fold increase after stromelysin digestion & Range & $n$ & Diagnosis \\
\hline & $y r$ & & & & \\
\hline A & 22-wk gestation & $>13172$ & & 1 & $\mathrm{C}$ \\
\hline B & 22-wk gestation & 979 & & 1 & $\mathrm{C}$ \\
\hline $\mathrm{C}$ & 19-wk gestation & $>2953$ & $2175-3730$ & 2 & $\mathrm{C}$ \\
\hline $\mathrm{D}$ & 20-wk gestation & $>2732$ & $2705-4221$ & 3 & $\mathrm{C}$ \\
\hline $\mathrm{E}$ & 23-wk gestation & $>4280$ & $3282-4465$ & 4 & $\mathrm{C}$ \\
\hline $\mathrm{F}$ & $30 \mathrm{~d}$ & ND & & & $\mathrm{C}$ \\
\hline G1 & 12 & 21.43 & $16.11-37.05$ & 9 & $\mathrm{C}$ \\
\hline $\mathrm{G} 2$ & 12 & 20.97 & $12.04-30.45$ & 8 & $\mathrm{C}$ \\
\hline $\mathrm{H} 1$ & 28 & 7.87 & $5.63-9.58$ & 8 & $\mathrm{C}$ \\
\hline H1 (femur) & 28 & 5.74 & $5.31-10.68$ & 7 & $\mathrm{C}$ \\
\hline $\mathrm{H} 2$ & 28 & 5.30 & $4.37-7.23$ & 7 & $\mathrm{C}$ \\
\hline H2 (femur) & 28 & 7.27 & $5.93-13.45$ & 6 & $\mathrm{C}$ \\
\hline I & 29 & 11.26 & 8.99-12.79 & 3 & $\mathrm{CT}$ \\
\hline J (right) & 30 & ND & & & $\mathrm{C}$ \\
\hline$J$ (left) & 30 & ND & & & $\mathrm{C}$ \\
\hline $\mathrm{K}$ & 40 & 9.30 & $8.80-9.80$ & 2 & $\mathrm{CT}$ \\
\hline $\mathrm{L}$ & 40 & 7.90 & & 1 & $\mathrm{C}$ \\
\hline M & 45 & ND & & & $\mathrm{C}$ \\
\hline $\mathrm{N}$ & 59 & 3.22 & & 1 & $\mathrm{C}$ \\
\hline $\mathrm{O}$ & 61 & 5.76 & $3.26-8.26$ & 2 & $\mathrm{CT}$ \\
\hline $\mathrm{P}$ & 62 & 9.99 & $5.48-24.91$ & 6 & $\mathrm{C}$ \\
\hline Q & 72 & 10.18 & $3.79-20.46$ & 7 & $\mathrm{C}$ \\
\hline $\mathrm{R}$ & 88 & 4.98 & & 1 & $\mathrm{C}$ \\
\hline 17 & 56 & 6.31 & $5.54-7.08$ & 2 & $\mathrm{RA}$ \\
\hline 4 & 59 & 2.14 & & 1 & OA \\
\hline 13 & 60 & 18.03 & $4.35-31.70$ & 2 & $\mathrm{OA}$ \\
\hline 12 & 64 & 7.18 & $5.90-8.45$ & 2 & $\mathrm{OA}$ \\
\hline 7 & 65 & 25.33 & & 1 & $\mathrm{OA}$ \\
\hline 9 & 65 & 9.38 & $8.95-9.81$ & 2 & OA \\
\hline 10 & 65 & 7.47 & $5.82-9.11$ & 2 & $\mathrm{OA}$ \\
\hline 1 & 66 & 3.01 & & 1 & $\mathrm{RA}$ \\
\hline 3 & 67 & 3.68 & & 1 & RA \\
\hline 16 & 69 & 23.82 & $10.40-40.50$ & 3 & OA \\
\hline 2 & 71 & 4.05 & & 1 & $\mathrm{RA}$ \\
\hline 11 & 76 & 7.14 & $5.43-8.33$ & 3 & OA \\
\hline 5 & 78 & 14.04 & & 1 & OA \\
\hline 6 & 78 & 8.57 & $5.18-18.00$ & 3 & RA \\
\hline 14 & 79 & 7.16 & & 1 & RA \\
\hline 18 & 82 & 3.14 & & 1 & OA \\
\hline 19 & 83 & 7.39 & & 1 & $\mathrm{OA}$ \\
\hline 8 & 84 & 4.57 & $4.31-7.28$ & 3 & $\mathrm{OA}$ \\
\hline 15 & 84 & 11.40 & $11.11-11.65$ & 2 & $\mathrm{OA}$ \\
\hline
\end{tabular}

Extracts of human knee joint cartilage (Diagnosis: C, control; CT, Control from tumor case) were prepared and the FVDIPEN neoepitope quantified by RIA. The samples were then exhaustively digested with stromelysin-1 to cleave all uncleaved, available aggrecan substrate and the FVDIPEN content again quantified using the RIA. Data are reported as fold increase after stromelysin digestion. For numbering and further information, see Table I.

10 median levels]) or RA samples $(0.185 \pm 0.114 \mathrm{pmol} / \mu \mathrm{g}$ hypro [ $n=6$ median levels]). Furthermore, the range of levels of the VDIPEN neoepitope relative to collagen in both the $\mathrm{OA}$ and RA samples was similar to those seen in the agematched control specimens (Table II). When the extracts of the OA samples were examined after stromelysin digestion, there were $\sim 8 \mathrm{~mol}$ of available aggrecan substrate for every mole of resident VDIPEN-positive G1 fragment (Table III). These levels were similar to those in the age-matched control cartilages.

The relative increase in the levels of VDIPEN neoepitope after stromelysin digestion in the specimens from patients with 


\section{pmol VDIPEN per $\mu \mathrm{g}$ OHpro after stromelysin digestion relative to uncleaved aggrecan substrate}

in Human Control Cartilage

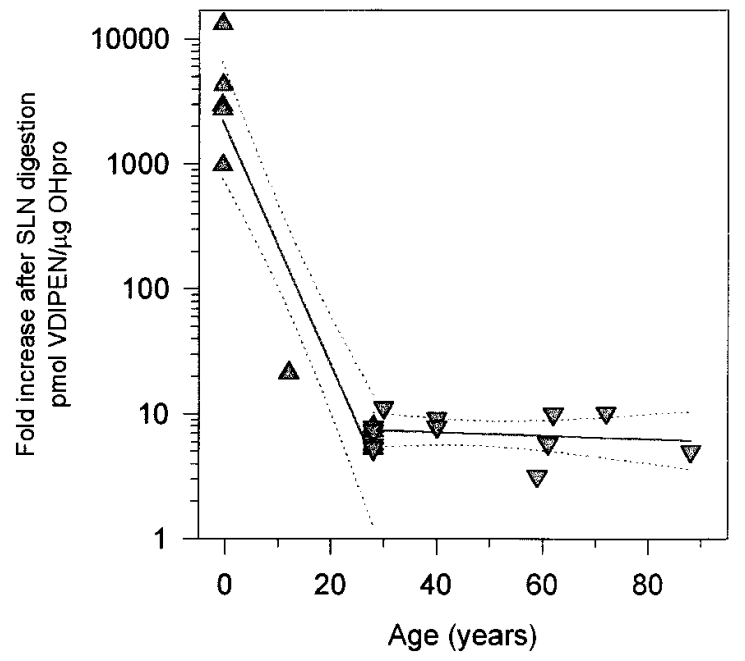

Figure 8. Increase in picomole of VDIPEN neoepitope per microgram hydroxyproline after stromelysin digestion of human joint cartilage from control patients with no history of joint disease. Results are expressed as fold increase in neopitope and plotted in relation to patient age. A first order regression line with $95 \%$ confidence interval (dotted lines) is plotted for the ages 0 to $28 \mathrm{yr}$ and $28-72 \mathrm{yr}$, respectively. Note logarithmic Y-axis.

RA (median increase of 5.2-fold after stromelysin digestion), was similar to that for both age-matched specimens from patients with $\mathrm{OA}$ and controls.

\section{Discussion}

We have shown, for the first time, that human articular cartilage contains two unique catabolic aggrecan G1 fragments, with structures consistent with MMP- and aggrecanase-generated core protein cleavage, respectively. The fragments were identified, characterized and localized by Western blotting, immunohistochemistry, and radioimmunoassay. Both types of fragments were found not only in cartilage from patients with $\mathrm{OA}$ and RA, but also in control cartilage obtained from individuals with no clinically diagnosed joint disease. Together the data suggest that both MMPs and aggrecanase play a role in turnover of human cartilage aggrecan.

Using $\mathrm{NH}_{2}$-terminal sequence analysis, it was previously reported that MMP-generated G1-fragments reside within human OA cartilage (11). We have extended these observations using a monospecific antiserum which recognizes MMP-cleaved aggrecan G1-fragments and have shown that such fragments can be isolated from human joint cartilage in both OA and RA. Furthermore, we have shown that these fragments are highly enriched in severely damaged, fibrillated areas of articular cartilage. The same fragments were, however, also found in cartilage from adult patients with no known joint disease. Similar to the distribution in the specimens from the patients with OA and RA, the neoepitope was enriched in the most severely damaged regions of the tissue.
When the VDIPEN neoepitope was quantified in cartilage from individuals with no known joint disease, the levels were significantly higher in adult than in young tissue, whether expressed relative to collagen content or relative to the amount of uncleaved aggrecan substrate. The concentration of VDIPEN neoepitope in adult joint cartilage was significant compared to the total available aggrecan substrate, representing some 15 $20 \%$ of the resident aggrecan molecules within the matrix. These data are consistent with the previously reported increase in aggrecan hyaluronan-binding region in cartilage with growth and maturation $(17,18)$. Our data show that at least one of these fragments is the MMP-generated G1-fragment.

We have also isolated NITEGE-positive, aggrecanasegenerated G1-fragments and characterized their distribution within joint cartilage from these same patients. Similar to the MMP-generated G1-fragment, the aggrecanase-generated fragments appear to be full length G1-fragments (23). Once quantitation of this fragment can be completed, it will be interesting to determine how much this G1-fragment contributes to the hyaluronan-binding G1-fragment pool which accumulates in cartilage with age. The distribution of the NITEGE neoepitope is similar to VDIPEN in most cases but in other cases the NITEGE neoepitope is present in regions where the VDIPEN neoepitope is undetectable. The isolation and tissue distribution of an aggrecanase-generated G1 fragment in human cartilage has not previously been described. The pattern of NITEGE neoepitope expression is similar in many ways to that previously reported for the distribution in the mouse collagen-induced arthritis model (37).

Rat chondrocytes can generate the NITEGE-positive G1 fragment when treated with retinoic acid in vitro (23), without any evidence of concomitant MMP-cleavage of the aggrecan molecule. It has also been reported that ARGSVIL-positive $(25,26)$ and FFGVG-positive (38) aggrecan fragments are found in joint fluids of patients with both OA and inflammatory arthritides. In this study we have shown that both NITEGEpositive and VDIPEN-positive G1 fragments are found within human articular cartilage. When these data are compiled to generate a model of cartilage aggrecan catabolism, a number of possibilities can exist (Fig. 9). First, it is possible that MMPs initially cleave aggrecan, leaving a G1-VDIPEN fragment and that aggrecanase further degrades the released large aggrecan molecule between $\mathrm{Asn}^{342}-\mathrm{Ala}^{374}$ as it diffuses out of the tissue. This would result in VDIPEN-positive G1 fragments remaining attached to hyaluronan in the cartilage with small FFGVGpositive interglobular domain fragments and larger ARGSVILpositive aggrecan fragments appearing in the joint fluid. This hypothesis is, however, not consistent with the presence of NITEGE-positive G1 fragments in the tissue. A second possibility is that aggrecanase cleaves aggrecan first and that MMPs further process (at least some of) the G1-fragments remaining within the cartilage. This would result in both ARGSVIL-positive aggrecan fragments and FFGVG-positive interglobular domain fragments in joint fluid, and both VDIPEN- and NITEGE-positive G1 fragments in the cartilage. The third possibility is that both proteolytic pathways may act independently and thus generate both VDIPEN- and NITEGE-positive G1-fragments in the cartilage and FFGVG-positive and ARGSVIL-positive aggrecan fragments in the joint fluid.

With the current data, it is most likely that either mechanism two or three is acting within the joint. Our observation that immunoreactivity for NITEGE is not abolished by diges- 

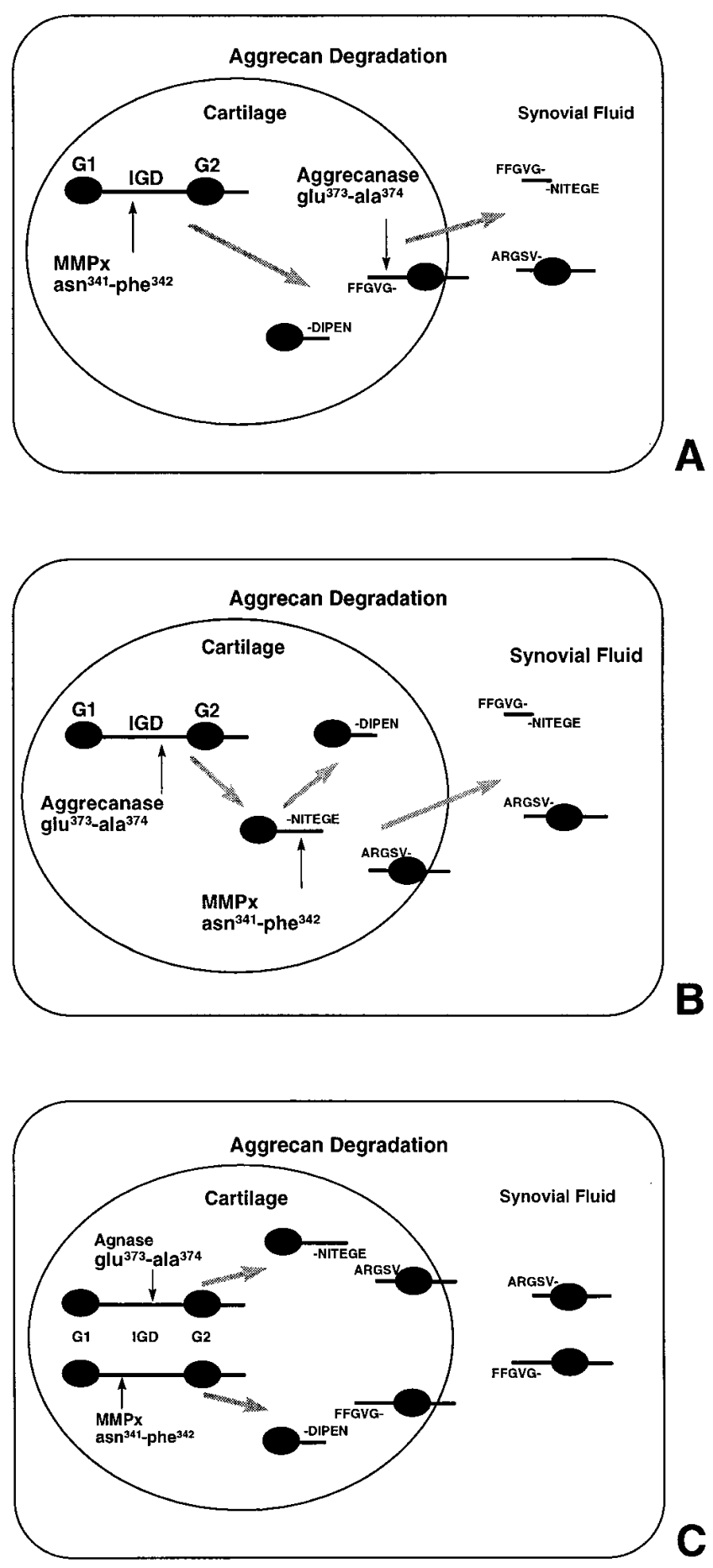

Figure 9. Hypothetical models for pathways of proteolytic breakdown of aggrecan in cartilage. (A) Primary cleavage of aggrecan interglobular domain $(I G D)$ by a classical matrix metalloprotease (MMPx), followed by aggrecanase (Agnase) cleavage of a 30-amino acid peptide from the liberated G2-containing fragment. Only VDIPEN neoepitope would be detected in matrix, while fragments with ARGSV or FFGVG amino termini would be present in joint fluid. (B) Primary cleavage of IGD by aggrecanase, followed by secondary cleavage by metalloproteinase activity. Depending on efficiency of second cleavage step, both NITEGE and VDIPEN neoepitopes may be detected in cartilage matrix, while large fragments with an amino terminus of ARGSV and a small 30-amino acid peptide with FFGVG- and -NITEGE termini will be detected in joint fluid. $(C)$ Aggrecanase and a classic matrix metalloproteinase activity tion of tissue sections with stromelysin-1, would perhaps suggest that the MMP- and aggrecanase-dependent pathways act independently, and argue in favor of mechanism three (Fig. 9C).

It has been shown by both in vivo and in vitro experiments that there are at least two pools of aggrecan surrounding chondrocytes which have different rates of turnover (39-41). In this study, we have shown that both the NITEGE and VDIPEN neoepitopes are generated, and in some cases, enriched in the immediate pericellular matrix. There is also neoepitope in regions far away from the chondrocyte. The intense staining immediately surrounding the cells suggests that both the aggrecanase enzyme and the MMPs are likely generated and or activated by the chondrocytes. The neoepitope-related reactivity also present in matrix further removed from the cells, on the other hand, suggests that the fragments may move away from the cells once generated or that the enzymes responsible for the aggrecan cleavage may be active within the interterritorial matrix. Since aggrecan molecules in the pericellular matrix appear to turn over more rapidly than those in the surrounding matrix (41), it would be interesting to examine the generation of these two different neoepitopes in vitro and determine if they turn over with different rates in these different matrix compartments.

Very limited immunostaining for NITEGE, and no immunostaining for VDIPEN was detected in normal mouse articular cartilage, but immunostaining for both epitopes was readily detected in the cartilage of mice with type II collagen-induced arthritis $(37,42)$. The data suggest, that in the normal articular cartilage of these animals there are few G1-fragments terminating in VDIPEN ${ }^{341}$ or NITEGE ${ }^{373}$, but that with increasing pathology there is a significant increase in both MMP- and aggrecanase-generated aggrecan cleavage.

Our present observations indicate that the content of MMP-generated aggrecan G1-fragments in human joint cartilage increases up to about 20-30 yr of age, and that the proportion (relative to both collagen and available aggrecan substrate) then remains constant with further increase in age. Moreover, the proportion of G1-VDIPEN in OA and RA joint cartilage, relative to collagen and to available aggrecan substrate, was about the same as in adult joint cartilage.

The observations on the occurrence of these molecular fragments in normal and pathologic human and animal tissues should be interpreted in the light of the fact that we can, by immunohistochemistry or by extraction of these human cartilage samples, detect only those fragments which remain in the tissue after being cleaved by MMP or aggrecanase. Aggrecan G1 fragments which diffuse into the joint fluid or are taken up by the chondrocytes escape our detection by these means. The aggrecan fragments detected here are thus the accrued result of a series of synthetic and catabolic events: aggrecan synthesis and secretion, proteolytic cleavage of the interglobular domain, and removal of the G1 fragment by chondrocyte uptake or diffusion (Fig. 10). The balance of these processes may differ be-

independently cleave aggrecan IGD. The G1-domains may remain bound to hyaluronan in matrix, while the remainder of the aggrecan molecules (including the G2-domain) are free to diffuse into joint fluid. Cleavage epitopes -NITEGE and -VDIPEN may be detected in tissue, while ARGSV- and FFGVG- may be detected in synovial fluid. 


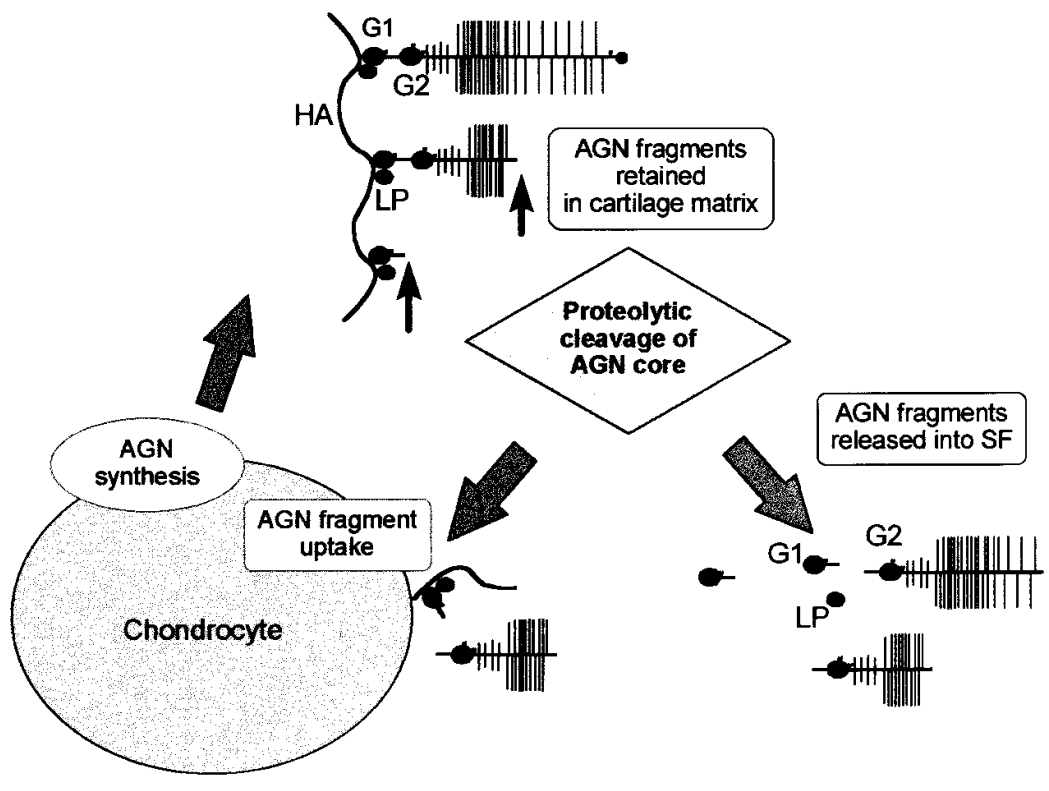

Figure 10. Schematic diagram of metabolic pathways of G1-containing fragments of joint cartilage aggrecan. The cartilage matrix content of aggrecan G1 fragments containing the carboxy termini of VDIPEN $^{341}$ or NITEGE ${ }^{373}$ is the result of $(a)$ the rate of production of aggrecan by the chondrocyte, $(b)$ the rates of aggrecan IGD cleavage by MMP or aggrecanase, $(c)$ the rate of chondrocyte uptake of the ternary complex of hyaluronan-link-G1 or free G1, and (d) the rate of diffusion out of the matrix of the ternary complex of hyaluronan-link-G1 or free G1. This assumes that there is no further $\mathrm{COOH}$-terminal processing of either fragment. $A G N$, aggrecan; $H A$, hyaluronan; $L P$, link protein. Small arrows, proteolytic cleavage of aggrecan core protein. Large arrows, pathways of addition or elimination of aggrecan or its cleavage products from matrix. tween tissues at different growth rates or ages, and with different degrees of pathology, and be influenced by changes in other matrix molecules which may interact with aggrecan. An example of such change is the increasing damage to type II collagen which has been demonstrated in aging and OA cartilage (43). It appears that these processes may reach a steady state in adult articular cartilage matrix, at least with regard to G1VDIPEN. Recently, VDIPEN fragments have been isolated from human OA joint fluids (Lark, M.W., and L.S. Lohmander, unpublished observations), suggesting that these fragments are indeed released from human articular cartilage. In support, it was shown that within $1 \mathrm{~h}$ after injection of stromelysin-1 into rabbit joints, $\sim 30 \%$ of the VDIPEN-positive G1 fragments are released into the joint fluid, suggesting that there is further processing of the HA-G1 complex resulting in release from the articular cartilage (44). In this context, it is interesting to note that the presence of aggrecan G1-fragments has been shown in joint fluids from patients with RA or OA $(45,46)$. Moreover, in vitro work on bovine joint cartilage has indicated that hyaluronan and aggrecan are catabolized at similar rates and it was suggested that at least part of the degradation involved internalization by the chondrocytes $(47,48)$. Examination of these events in a closed experimental system, such as cartilage explants or chondrocytes grown in alginate in vitro, may help to clarify some of these questions.

The results of our study indicate that both MMPs and aggrecanase play roles in the degradation of human cartilage aggrecan in normal cartilage as well as in osteoarthritic and rheumatoid cartilage. We find it interesting that similar aggrecan degradation mechanisms may be active in osteoarthritis and rheumatoid arthritis, in spite of the contrasting pathological and clinical features of these two conditions. It will be important to compare the distribution and turnover of the aggrecan G1 fragments carrying the VDIPEN and NITEGE neoepitopes in samples from patients with various joint diseases, to try to unravel the seemingly complex controls regulating aggrecan metabolism within the joint. Such information may be helpful in efforts to intervene with cartilage destruction in joint disease.

\section{Acknowledgments}

L.S. Lohmander was supported by the Swedish Medical Research Council, the King Gustaf V 80-year Birthday Fund, the Kock and the Zoega Foundations, the Medical Faculty of Lund and Lund University Hospital, and Merck Research Laboratories.

\section{References}

1. Dean, D.D., J. Martel-Pelletier, J.-P. Pelletier, D.S. Howell, and J.F.J. Woessner. 1989. Evidence for metalloproteinase and metalloproteinase inhibitor imbalance in human osteoarthritic cartilage. J. Clin. Invest. 84:678-685.

2. Gravallese, E.M, J.M. Darling, A.L. Ladd, J.N. Katz, and L.H. Glimcher. 1991. In situ hybridization studies of stromelysin and collagenase expression in rheumatoid synovium. Arthritis Rheum. 34:1076-1084.

3. McCachren, S.S. 1991. Expression of metalloproteinases and metalloproteinase inhibitor in human arthritic synovium. Arthritis Rheum. 34:1085-1093.

4. Firestein, G.S., M.M. Paine, and B.H. Littman. 1991. Gene expression (collagenase, tissue inhibitor of metalloproteinases, complement, and HLADR) in rheumatoid arthritis and osteoarthritis synovium. Quantitative analysis and effect of intraarticular corticosteroids. Arthritis Rheum. 34:1094-1105.

5. Walakovits, L.A., V.L. Moore, N. Bhardwaj, G.S. Gallick, and M.W. Lark. 1991. Detection of high levels of stromelysin and collagenase in synovial fluid from patients with rheumatoid arthritis and post-traumatic knee injury. Arthritis Rheum. 35:35-42.

6. Nguyen, Q., J.S. Mort, and P.J. Roughley. 1992. Preferential mRNA expression of prostromelysin relative to procollagenase and in situ localization in human articular cartilage. J. Clin. Invest. 89:1189-1197.

7. Lohmander, L.S., L.A. Hoerrner, and M.W. Lark. 1993. Metalloproteinases, tissue inhibitor and proteoglycan fragments in knee synovial fluid in human osteoarthritis. Arthritis Rheum. 36:181-189.

8. Wolfe, G.C., K.L. MacNaul, F.F. Buechel, J. McDonnell, L.A. Hoerrner, M.W. Lark, V.L. Moore, and N.I. Hutchinson. 1993. Differential in vivo expression of collagenase messenger RNA in synovium and cartilage. Quantitative comparison with stromelysin messenger RNA levels in human rheumatoid arthritis and osteoarthritis patients and in two animal models of acute inflammatory arthritis. Arthritis Rheum. 36:1540-1547.

9. Lohmander, L.S., L.A. Hoerrner, and M.W. Lark. 1995. Increased concentrations of collagenase (MMP-1) in joint fluid after knee injury and in osteoarthritis. Trans. Orthop. Res. Soc. 20:9. (Abstr.)

10. Nguyen, Q., G. Murphy, P.J. Roughley, and J.S. Mort. 1989. Degradation of proteoglycan aggregate by a cartilage metalloproteinase. Evidence for the involvement of stromelysin in the generation of link protein heterogeneity in situ. Biochem. J. 259:61-67.

11. Flannery, C.R., M.W. Lark, and J.D. Sandy. 1992. Identification of a stromelysin cleavage site within the interglobular domain of human aggrecan: evidence for proteolysis at this site in vivo in human articular cartilage. J. Biol. Chem. 267:1008-1014.

12. Fosang, A.J., K. Last, V. Knäuper, P.J. Neame, G. Murphy, T.E. Har- 
dingham, H. Tschesche, and J.A. Hamilton. 1993. Fibroblast and neutrophil collagenases cleave at two sites in the cartilage aggrecan interglobular domain. Biochem. J. 295:273-276.

13. Fosang, A.J., P.J. Neame, T.E. Hardingham, G. Murphy, and J.A. Hamilton. 1991. Cleavage of cartilage proteoglycan between G1 and G2 domains by stromelysin. J. Biol. Chem. 266:15579-15582

14. Wu, J.-J., M.W. Lark, L.E. Chun, and D.R. Eyre. 1991. Sites of stromelysin cleavage in collagen types II, IX, X, and XI of cartilage. J. Biol. Chem. 266: $5625-5628$.

15. Liu, J.J., D. Cassidy, A. Allan, P.J. Neame, J.S. Mort, and P.J. Roughley. 1992. Link protein shows species variation in its susceptibility to proteolysis. $J$. Orthop Res. 10:621-630.

16. Hollander, A.P., T.F. Heathfield, C. Webber, Y. Iwata, R. Bourne, C. Rorabeck, and A.R. Poole. 1994. Increased damage to type II collagen in osteoarthritic cartilage detected by a new immunoassay. J. Clin. Invest. 93:17221732 .

17. Roughley, P.J., R.J. White, and A.R. Poole. 1985. Identification of a hyaluronic acid-binding protein that interferes with the preparation of high-buoyant density proteoglycan aggregates from adult human articular cartilage. Biochem. J. 231:129-138.

18. Bayliss, M.T., M.W.A. Holmes, and H. Muir. 1989. Age-related changes in the stoichiometry of binding region, link protein and hyaluronic acid in human articular cartilage. Trans. Orthop. Res. Soc. 14:32. (Abstr.)

19. Sandy, J.D., P.J. Neame, R.E. Boynton, and C.R. Flannery. 1991. Catabolism of aggrecan in cartilage explants. Identification of a major cleavage site within the interglobular domain. J. Biol. Chem. 266:8683-8695.

20. Ilic, M.Z., C.H. Handley, H.C. Robinson, and M.T. Mok. 1992. Mechanism of catabolism of aggrecan by articular cartilage. Arch. Biochem. Biophys. 294:115-122.

21. Loulakis, P., A. Shrikande, G. Davis, and C.A. Maniglia. 1992. N-terminal sequence of proteoglycan fragments isolated from medium of interleukin-1treated articular-cartilage cultures. Biochem. J. 284:589-593.

22. Plaas, A.H., and J.D. Sandy. 1993. A cartilage explant system for studies on aggrecan structure, biosynthesis and catabolism in discrete zones of the mammalian growth plate. Matrix. 13:135-147.

23. Lark, M.W., J.T. Gordy, J.R. Weidner, J. Ayala, J.H. Kimura, H.R. Williams, R.A. Mumford, C.R. Flannery, S.S. Carlson, M. Iwata, and J.D. Sandy. 1995. Cell-mediated catabolism of aggrecan. Evidence that cleavage at the "aggrecanase" site $\left(\mathrm{Glu}^{373}-\mathrm{Ala}^{374}\right)$ is a primary event in proteolysis of the interglobular domain. J. Biol. Chem. 270:2550-2556.

24. Hughes, C.E., B. Caterson, A.J. Fosang, P.J. Roughley, and J.S. Mort. 1995. Monoclonal antibodies that specifically recognize neoepitope sequences generated by 'aggrecanase' and matrix metalloproteinase cleavage of aggrecan: application to catabolism in situ and in vitro. Biochem. J. 305:799-804.

25. Lohmander, L.S., P. Neame, and J.D. Sandy. 1993. The structure of aggrecan fragments in human synovial fluid: Evidence that aggrecanase mediates cartilage degradation in inflammatory joint disease, joint injury and osteoarthritis. Arthritis Rheum. 36:1214-1222.

26. Sandy, J.D. C.R. Flannery, P.J. Neame, and L.S. Lohmander. 1992. The structure of aggrecan fragments in human synovial fluid: Evidence for the involvement in osteoarthritis of a novel proteinase which cleaves the glu 373-ala 374 bond of the interglobular domain. J. Clin. Invest. 89:1512-1516.

27. Fosang, A.J., K. Last, P.J. Neame, G. Murphy, V. Knäuper, H. Tschesche, C. Hughes, B. Caterson, and T.E. Hardingham. 1994. Neutrophil collagenase (MMP-8) cleaves at the aggrecanase site E373-A-374 in the interglobular domain of cartilage aggrecan. Biochem. J. 304:347-351.

28. Lark, M.W., H. Williams, L.A. Hoerrner, J.R. Weidner, J.M. Ayala, C.F. Harper, A. Christen, J. Olszewski, Z. Konteatis, R. Webber, et al. 1995. Quantification of a matrix metalloproteinase-generated aggrecan G1 fragment using monospecific anti-peptide serum. Biochem. J. 307:245-252.

29. Doege, K.J., M. Sasaki, T. Kimura, and Y. Yamada. 1991. Complete coding sequence and deduced primary structure of the human cartilage large aggregating proteoglycan, aggrecan. J. Biol. Chem. 266:894-902.

30. Hascall, V.C., and J.H. Kimura. 1982. Proteoglycans: isolation and characterization. Methods Enzymol. 82:769-800.
31. Woessner, J.F., Jr. 1961. The determination of hydroxyproline in tissue and protein samples containing small proportions of this imino acid. Arch. Biochem. Biophys. 93:440-447.

32. MacLean, I.W., and P.K. Nakane. 1974. Periodate-lysine-paraformaldehyde fixative. A new fixation for immunoelectron microscopy. J. Histochem. Cytochem. 35:647-655.

33. Shu, S., G. Ju, and L. Fan. 1988. The glucose oxidase-DAB nickel method in peroxidase histochemistry of the nervous system. Neurosci. Lett. 85: 169-171.

34. Mankin, H.J., H. Dorfman, L. Lippiello, and A. Zarins. 1971. Biochemical and metabolic abnormalities in articular cartilage from osteo-arthritic human hips. II. Correlation of morphology with biochemical and metabolic data. J. Bone Jt. Surg. (Am.) Vol. 53:523-537.

35. Lark, M.W., L.A. Walakovits, T.K. Shah, J. Vanmiddlesworth, P.M Cameron, and T.-Y. Lin. 1990. Production and purification of prostromelysin and procollagenase from IL-1 beta-stimulated human gingival fibroblasts. Connect. Tissue Res. 25:49-65.

36. Teahan, J., R. Harrison, M. Izquierdo, and R.L. Stein. 1989. Substrate specificity of human fibroblast stromelysin. Hydrolysis of substance P and its analogues. Biochemistry. 28:8497-8501.

37. Singer, I.I., S. Scott, D.W. Kawka, E.K. Bayne, S.A. Donatelli, J.R. Weidner, H.R. Williams, R.A. Mumford, M.W. Lark, J. McDonnell, et al. 1995. Aggrecanase and metalloproteinase-specific aggrecan neoepitopes are induced in the articular cartilage of mice with collagen II arthritis. Trans. Orthop. Res. Soc. 20:330.

38. Fosang, A.J., K. Last, and R.A. Maciewicz. 1996. Aggrecan is degraded by matrix metalloproteinases in human arthritis. Evidence that matrix metalloproteinases and aggrecanase activities can be independent. J. Clin. Invest. 98: 2292-2299.

39. Lohmander, S. 1977. Turnover of proteoglycans in guinea pig costal cartilage. Arch. Biochem. Biophys. 180:93-101.

40. Sandy, J.D. 1992. Extracellular metabolism of aggrecan. In Articular Cartilage and Osteoarthritis. K.E. Kuettner, R. Schleyerbach, J.G. Peyron, and V.C. Hascall, editors. Raven Press, Ltd., New York. 21-33.

41. Mok, S.S., K. Masuda, H.J. Häuselmann, M.B. Aydelotte and E.J.-M.A. Thonar. 1994. Aggrecan synthesized by mature chondrocytes suspended in alginate. Identification of two distinct metabolic pools. J. Biol. Chem. 269:3302133027.

42. Singer, I.I., D.W. Kawka, E.K. Bayne, S.A. Donatelli, J.R. Weidner, H.R. Williams, J.M Ayala, R.A. Mumford, M.W. Lark, T.T. Glant, et al. 1995. VDIPEN, a metalloproteinase-generated neoepitope, is induced and immunolocalized in articular cartilage during inflammatory arthritis. J. Clin. Invest. 95:2178-2186.

43. Hollander, A.P., I. Pidoux, A. Reiner, C. Rorabeck, R. Bourne, and A.R. Poole. 1995. Damage to type II collagen in aging and osteoarthritis starts at the articular surface, originates around chondrocytes, and extends into the cartilage with progressive degeneration. J. Clin. Invest. 96:2859-2869.

44. Bayne, E.K, K.L. MacNaul, S.A. Donatelli, A. Christen, P.R. Griffin, L.A. Hoerrner, J.R. Calaycay, J.M. Ayala, K. Chapman, W. Hagmann, et al. 1995. Use of an antibody against the matrix metalloproteinase-generated aggrecan neoepitope FVDIPEN-COOH to assess the effects of stromelysin in a rabbit model of cartilage degradation. Arthritis Rheum. 38:1400-1409.

45. Saxne, T., and D. Heinegård. 1992. Synovial fluid analysis of two groups of proteoglycan epitopes distinguishes early and late cartilage lesions. Arthritis Rheum. 35:385-390.

46. Sandy, J.D., A.H.K. Plaas, and T.J. Koob. 1995. Pathways of aggrecan processing in joint tissues-implications for disease mechanism and monitoring. Acta Orthop. Scand. 66 (Suppl. 266):26-32.

47. Morales, T.I., and V.C. Hascall. 1988. Correlated metabolism of proteoglycans and hyaluronic acid in bovine articular cartilage organ cultures. $J$. Biol. Chem. 263:3632-3638.

48. Morales, T.I., and V.C. Hascall. 1989. Effects of interleukins and lipopolysaccharides on protein and carbohydrate metabolism in bovine articular cartilage organ cultures. Connect. Tissue Res. 19:255-275. 